\title{
Discrete canonical system and non-Abelian Toda lattice: Bäcklund-Darboux transformation, Weyl functions, and explicit solutions
}

\author{
A.L. Sakhnovich
}

Branch of Hydroacoustics, Marine Institute of Hydrophysics, National Academy of Sciences of Ukraine

e-mail addresses: al_sakhnov@yahoo.com

Short title. Discrete canonical system

\begin{abstract}
A version of the iterated Bäcklund-Darboux transformation, where Darboux matrix takes a form of the transfer matrix function from the system theory, is constructed for the discrete canonical system and Non-Abelian Toda lattice. Results on the transformations of the Weyl functions, insertion of the eigenvalues, and construction of the bound states are obtained. A wide class of the explicit solutions is given. An application to the semi-infinite block Jacobi matrices is treated.
\end{abstract}

\section{Introduction}

The matrix discrete canonical system (DCS) has the form [32, [41]:

$$
w(k, l)-w(k-1, l)=i l J H_{k} w(k-1, l) \quad(k \geq 1),
$$


where $H_{k}$ are $m \times m$ matrices (the set $\left\{H_{k}\right\}$ we sometimes call Hamiltonian), and

$$
J=J^{*}=J^{-1}, \quad H_{k}=H_{k}^{*}, \quad H_{k} J H_{k}=0 .
$$

This system is a discretization of the classical canonical system [10, 20, 42]:

$$
\frac{d}{d x} w(x, l)=i l J H(x) w(x, l) \quad(H(x) \geq 0) .
$$

DCS is equivalent to an important subclass of the well-known Jacobi systems 32 and it is an auxiliary system for the matrix Toda lattice (MTL) studied in [41]:

$$
\left\{\begin{array}{c}
Q^{\prime}(k, t)=C(k+1, t) C(k, t)^{-1}-C(k, t) C(k-1, t)^{-1} \\
C^{\prime}(k, t)=Q(k, t) C(k, t) .
\end{array}\right.
$$

Here $C(k, t)$ and $Q(k, t)$ are $p \times p(p=m / 2)$ matrix functions, $Q^{\prime}(t)=\frac{d}{d t} Q(t)$, and

$$
C(k, t) Q(k, t)^{*}-Q(k, t) C(k, t)=0, \quad C(k, t)=C(k, t)^{*}, \quad \operatorname{det} C(k, t) \neq 0 .
$$

If system (1.3) is valid, $C(k, t)>0$ and $p=1$, then the first relation in (1.4) holds automatically and the functions $u(k, t)=-\ln C(k, t)$ satisfy the classical Toda lattice

$$
\frac{d^{2}}{d t^{2}} u(k, t)=\exp [u(k-1, t)-u(k, t)]-\exp [u(k, t)-u(k+1, t)] .
$$

The Non-Abelian Toda lattice (NTL) was introduced by Polyakov [23] as a discretization of the principal chiral field equation and coincides with system (1.3) without additional condition (1.4). When the auxiliary Jacobi matrices are used NTL is presented sometimes [16] in the form

$$
\mathcal{A}_{k}^{\prime}(t)=\mathcal{B}_{k+1}(t) \mathcal{A}_{k}(t)-\mathcal{A}_{k}(t) \mathcal{B}_{k}(t), \quad \mathcal{B}_{k}^{\prime}(t)=\mathcal{A}_{k}(t)-\mathcal{A}_{k-1}(t) \quad(k>0)
$$

The equivalence between systems (1.3) and (1.6) is given by the relations

$$
\mathcal{A}_{k}(t)=C(k+1, t) C(k, t)^{-1}, \quad \mathcal{B}_{k}(t)=Q_{k}(t),
$$


and vice versa:

$$
\begin{gathered}
Q_{k}(t)=\mathcal{B}_{k}(t), \quad C(k, t)=\left(\prod_{l=0}^{k-1} \mathcal{A}_{l}(t)\right) C(0, t), \\
C^{\prime}(0, t)=\mathcal{A}_{0}(t)^{-1}\left(Q(1, t)-\mathcal{A}_{0}^{\prime}(t) \mathcal{A}_{0}(t)^{-1}\right) \mathcal{A}_{0}(t) C(0, t) .
\end{gathered}
$$

Interesting spectral results for the Toda lattice and its MTL and NTL generalizations have been obtained in [3, 4, 6, 15, 21, 24, 41, 44, 46] (see also references), though various related spectral problems are still to be solved.

We shall construct a Bäcklund-Darboux transformation (BDT) for the DCS (1.1), MTL and NTL. The BDTs are widely used in the spectral and in the integrable nonlinear equations theories, and the construction of the explicit solutions is one of the important applications. Numerous results and literature on BDTs are contained in [2, 7, 17, 28, 29, 31, 47]. In spite of various important results (see [2, 5, 8, 12, 25, 29, 45] and references therein) the BDTs for the discrete equations are more complicated and less studied than in the continuous case 2]. An interesting modification of the BDT have been suggested by V.A. Marchenko. Some developments and applications to the explicit solutions of the Toda lattice and non-Abelian two-dimensional Toda lattice one can find in [43] and [13], respectively. The version of the BDT that we are going to apply (GBDT in the terminology of [36]) was initially developed in [33, 34] (see the case of the canonical system in 35] and more references and applications in [19, 36]-[39]). The Darboux matrix in this approach is presented as the transfer matrix function, which is a well known tool in system theory 22]. Transfer matrix function of the form

$$
w_{A}(l)=I_{m}-\Pi(2)^{*} S^{-1}\left(A(1)-l I_{n}\right)^{-1} \Pi(1) \quad\left(A(1) S-S A(2)=\Pi(1) \Pi(2)^{*}\right),
$$

that we are going to use, was introduced by L. Sakhnovich in the context of his method of operator identities [40, 41]. Representation (1.10) takes roots in the Livšic-Brodskii characteristic matrix function [27.

GBDT for the discrete canonical system is constructed in Section 2. The results on the corresponding to GBDT transformations of the Weyl functions and Theorem 3.5 on the insertion of the eigenvalues and construction of the bound states are given in Section 3. GBDT for the matrix Toda lattice 
is constructed in Section 4 and its modification for the Non-Abelian Toda lattice is discussed in Section 6. A wide class of the explicit solutions of the Non-Abelian Toda lattice, the corresponding fundamental solutions of the discrete canonical systems, and evolution of the Weyl functions are described in Section 5. Appendix is dedicated to the analog of Theorem 3.5 for the block Jacobi matrices.

\section{Discrete canonical system: GBDT and Weyl functions}

To introduce the GBDT of the discrete canonical system suppose that system (1.1), (1.2) is given and fix an integer parameter $n>0$ and three parameter matrices: $n \times n$ matrices $A$ and $S_{0}$ and $n \times m$ matrix $\Pi_{0}$ satisfying the matrix identity

$$
A S_{0}-S_{0} A^{*}=i \Pi_{0} J \Pi_{0}^{*} \quad\left(S_{0}=S_{0}^{*}\right) .
$$

Then the sets of matrices $\Pi_{k}$ and $S_{k}$ are defined by the equalities

$$
\begin{gathered}
\Pi_{k}=\Pi_{k-1}-i A \Pi_{k-1} J H_{k} \quad(k \geq 1), \\
S_{k}=S_{k-1}+\Pi_{k-1} J H_{k} J \Pi_{k-1}^{*} \quad(k \geq 1) .
\end{gathered}
$$

System (2.2) is obtained from the system dual to (1.1) via the substitution

of the spectral parameter $l$ by the generalized eigenvalue - matrix $A$. System (2.3) is chosen so that the identities

$$
A S_{k}-S_{k} A^{*}=i \Pi_{k} J \Pi_{k}^{*} \quad(k \geq 0)
$$

hold. Indeed, in view of (2.3) we have

$$
A S_{k}-S_{k} A^{*}=A S_{k-1}-S_{k-1} A^{*}+A \Pi_{k-1} J H_{k} J \Pi_{k-1}^{*}-\Pi_{k-1} J H_{k} J \Pi_{k-1}^{*} A^{*} .
$$

Taking into account (1.2) and (2.2) we rewrite the equality above:

$$
A S_{k}-S_{k} A^{*}=A S_{k-1}-S_{k-1} A^{*}+i\left(\Pi_{k} J \Pi_{k}^{*}-\Pi_{k-1} J \Pi_{k-1}^{*}\right) .
$$


Formulas (2.1) and (2.5) yield identities (2.4). Define now a transfer matrix function $w_{A}(k, l)(l \notin \sigma(A), \sigma$ means spectrum) in the form (1.10):

$$
w_{A}(k, l)=I_{m}-i J \Pi_{k}^{*} S_{k}^{-1}\left(A-l I_{n}\right)^{-1} \Pi_{k} .
$$

Theorem 2.1 Let matrix function w satisfy initial DCS (1.1), (1.2). Choose integer $n>0$ and three parameter matrices: $n \times n$ matrices $A$ and $S_{0}$ and $n \times m$ matrix $\Pi_{0}$ such that (2.1) holds. Define matrices $\left\{\Pi_{k}\right\}$ and $\left\{S_{k}\right\}(k>$ 0) via (2.2) and (2.3), and suppose det $S_{k} \neq 0(0 \leq k \leq K)$. Choose a Junitary $m \times m$ matrix $w_{0}(0)$, i.e., choose $w_{0}(0)$ such that $w_{0}(0) J w_{0}(0)^{*}=J$, and define matrices $w_{0}(k)(0<k \leq K)$ by the relations

$$
w_{0}(k)=\left(I_{m}-J \Pi_{k}^{*} S_{k}^{-1} \Pi_{k} J H_{k}+J H_{k} J \Pi_{k-1}^{*} S_{k-1}^{-1} \Pi_{k-1}\right) w_{0}(k-1) .
$$

Then a solution $\widetilde{w}(k, l) \quad(0<k \leq K)$ of the transformed DCS

$$
\widetilde{w}(k, l)-\widetilde{w}(k-1, l)=i l J \widetilde{H}_{k} \widetilde{w}(k-1, l),
$$

where

$$
\begin{gathered}
\widetilde{H}_{k}=w_{0}(k)^{*}\left(H_{k}+H_{k} J \Pi_{k-1}^{*} S_{k-1}^{-1} \Pi_{k-1} J H_{k}\right) w_{0}(k), \\
\widetilde{H}_{k} J \widetilde{H}_{k}=0,
\end{gathered}
$$

is given by the formulas

$$
\widetilde{w}(k, l)=v(k, l) w(k, l), \quad v(k, l)=w_{0}(k)^{-1} w_{A}(k, l) .
$$

Moreover if $H_{k} \geq 0(0<k \leq K)$ and $S_{0}>0$, then $S_{k}>0$ and $\widetilde{H}_{k} \geq 0$ also.

System (2.8) is the GBDT of the initial system (1.1). Its Hamiltonian $\widetilde{H}_{k}$ and fundamental solution are expressed explicitly in terms of the Hamiltonian and fundamental solution of the initial system (see formulas (2.9) and (2.11)), which is the characteristic property of the BDT. GBDT (2.8) is determined by the parameter matrices $A, S_{0}$, and $\Pi_{0}$, satisfying identity (2.1).

Pr o of of Theorem 2.1. From (2.3) it follows easily that

$$
S_{k}^{-1}-S_{k-1}^{-1}=-S_{k}^{-1} \Pi_{k-1} J H_{k} J \Pi_{k-1}^{*} S_{k-1}^{-1} .
$$


In view of (2.12) we get

$$
\Pi_{k}^{*} S_{k}^{-1}-\Pi_{k-1}^{*} S_{k-1}^{-1}=\Pi_{k}^{*} S_{k-1}^{-1}-\Pi_{k}^{*} S_{k}^{-1} \Pi_{k-1} J H_{k} J \Pi_{k-1}^{*} S_{k-1}^{-1}-\Pi_{k-1}^{*} S_{k-1}^{-1} .
$$

Using (2.2) we rewrite this equality as

$$
\Pi_{k}^{*} S_{k}^{-1}-\Pi_{k-1}^{*} S_{k-1}^{-1}=i H_{k} J \Pi_{k-1}^{*} A^{*} S_{k-1}^{-1}-\Pi_{k}^{*} S_{k}^{-1} \Pi_{k-1} J H_{k} J \Pi_{k-1}^{*} S_{k-1}^{-1} .
$$

By (2.4) we have $A^{*} S_{k-1}^{-1}=S_{k-1}^{-1} A-i S_{k-1}^{-1} \Pi_{k-1} J \Pi_{k-1}^{*} S_{k-1}^{-1}$ and so equality (2.13) takes the form

$$
\begin{gathered}
\Pi_{k}^{*} S_{k}^{-1}-\Pi_{k-1}^{*} S_{k-1}^{-1}=i H_{k} J \Pi_{k-1}^{*} S_{k-1}^{-1} A+ \\
+\left(H_{k} J \Pi_{k-1}^{*} S_{k-1}^{-1} \Pi_{k-1} J-\Pi_{k}^{*} S_{k}^{-1} \Pi_{k-1} J H_{k} J\right) \Pi_{k-1}^{*} S_{k-1}^{-1} .
\end{gathered}
$$

Now we can derive the crucial equality

$$
\begin{gathered}
Z(l):=w_{A}(k, l)\left(I_{m}+i l J H_{k}\right)= \\
\left(I_{m}+i l J H_{k}-J \Pi_{k}^{*} S_{k}^{-1} \Pi_{k} J H_{k}+J H_{k} J \Pi_{k-1}^{*} S_{k-1}^{-1} \Pi_{k-1}\right) w_{A}(k-1, l) .
\end{gathered}
$$

Indeed, from (2.6), (2.2) and (1.2) it follows that

$$
\begin{gathered}
Z(l)=\left(I_{m}-i J \Pi_{k}^{*} S_{k}^{-1}\left(A-l I_{n}\right)^{-1} \Pi_{k-1}\right)\left(I_{m}+i l J H_{k}\right) \\
-J \Pi_{k}^{*} S_{k}^{-1}\left(A-l I_{n}\right)^{-1} A \Pi_{k-1} J H_{k} .
\end{gathered}
$$

Rewriting $A$ as $\left(A-l I_{n}\right)+l I_{n}$ we obtain

$$
Z(l)=I_{m}+i l J H_{k}-i J \Pi_{k}^{*} S_{k}^{-1}\left(A-l I_{n}\right)^{-1} \Pi_{k-1}-J \Pi_{k}^{*} S_{k}^{-1} \Pi_{k-1} J H_{k} .
$$

In view of (2.14) equality (2.17) yields

$$
\begin{gathered}
Z(l)=i l J H_{k}+w_{A}(k-1, l)+J H_{k} J \Pi_{k-1}^{*} S_{k-1}^{-1} A\left(A-l I_{n}\right)^{-1} \Pi_{k-1} \\
-i J\left(H_{k} J \Pi_{k-1}^{*} S_{k-1}^{-1} \Pi_{k-1} J-\Pi_{k}^{*} S_{k}^{-1} \Pi_{k-1} J H_{k} J\right) \Pi_{k-1}^{*} S_{k-1}^{-1}\left(A-l I_{n}\right)^{-1} \Pi_{k-1}
\end{gathered}
$$




$$
-J \Pi_{k}^{*} S_{k}^{-1} \Pi_{k-1} J H_{k} .
$$

By definition (2.6) and substitution $A=\left(A-l I_{n}\right)+l I_{n}$ we derive now

$$
Z(l)=\left(I_{m}+i l J H_{k}-J \Pi_{k}^{*} S_{k}^{-1} \Pi_{k-1} J H_{k}+J H_{k} J \Pi_{k-1}^{*} S_{k-1}^{-1} \Pi_{k-1}\right) w_{A}(k-1, l) .
$$

By (1.2), (2.2) and (2.19) formula (2.15) is immediate.

Now in view of (1.1) and (2.15) one gets

$$
\begin{aligned}
w_{A}(k, l) w(k, l)=\left(I_{m}+\right. & \left.i l J H_{k}-J \Pi_{k}^{*} S_{k}^{-1} \Pi_{k} J H_{k}+J H_{k} J \Pi_{k-1}^{*} S_{k-1}^{-1} \Pi_{k-1}\right) \times \\
\times & \times w_{A}(k-1, l) w(k-1, l) .
\end{aligned}
$$

According to (2.20) to prove that $\widetilde{w}$ of the form (2.11) satisfies (2.8) it remains to show that

$$
\begin{gathered}
I_{m}+i l J \widetilde{H}_{k}= \\
w_{0}(k)^{-1}\left(I_{m}+i l J H_{k}-J \Pi_{k}^{*} S_{k}^{-1} \Pi_{k-1} J H_{k}+J H_{k} J \Pi_{k-1}^{*} S_{k-1}^{-1} \Pi_{k-1}\right) w_{0}(k-1) .
\end{gathered}
$$

By the definition (2.7) formula (2.21) is equivalent to the relation

$$
J \widetilde{H}_{k}=w_{0}(k)^{-1} J H_{k} w_{0}(k-1) .
$$

It follows [40, 41] from (2.4) and (2.6) that

$$
w_{A}(k, l) J w_{A}(k, \bar{l})^{*}=J \quad(k \geq 0) .
$$

By (1.2) it is immediate that

$$
\left(I_{m}+i l J H_{k}\right) J\left(I_{m}+i l J H_{k}\right)^{*}=J \quad(l \in \mathbb{R}) .
$$

According to (2.15), (2.23) and (2.24) matrices

$$
I_{m}-J \Pi_{k}^{*} S_{k}^{-1} \Pi_{k} J H_{k}+J H_{k} J \Pi_{k-1}^{*} S_{k-1}^{-1} \Pi_{k-1}
$$

are $J$-unitary. Hence, taking into account formula (2.7), we obtain

$$
w_{0}(k) J w_{0}(k)^{*}=J
$$




$$
w_{0}(k-1)=J\left(I_{m}-J \Pi_{k}^{*} S_{k}^{-1} \Pi_{k} J H_{k}+J H_{k} J \Pi_{k-1}^{*} S_{k-1}^{-1} \Pi_{k-1}\right)^{*} J w_{0}(k) .
$$

Finally in view of the formulas (1.2), (2.7), (2.9), and (2.25) we derive

$$
\begin{gathered}
J \widetilde{H}_{k}=J w_{0}(k)^{*}\left(H_{k}+H_{k} J \Pi_{k-1}^{*} S_{k-1}^{-1} \Pi_{k-1} J H_{k}\right) w_{0}(k)= \\
w_{0}(k)^{-1} J H_{k} J\left(I_{m}+\Pi_{k-1}^{*} S_{k-1}^{-1} \Pi_{k-1} J H_{k} J-H_{k} J \Pi_{k}^{*} S_{k}^{-1} \Pi_{k} J\right) J w_{0}(k) .
\end{gathered}
$$

In view of (2.26) and (2.27) the equality (2.22) is true, and so (2.8) is satisfied. Equalities (2.10) follow from (2.9) and (2.25). The last statement of the theorem is immediate from (2.3) and (2.9).

Remark 2.2 When $A$ is invertible and $w_{0}(0)=w_{A}(0,0)$ then by (2.7) and (2.15) we have $w_{0}(k)=w_{A}(k, 0)$ for all $k \geq 0$.

Remark 2.3 Formula (2.23) is a particular case of a more general relation that follows [40, 41] from 20.4) and [2.6):

$$
w_{A}(k, l)^{*} J w_{A}(k, \mu)=J+i(\bar{l}-\mu) \Pi_{k}^{*}\left(A^{*}-\bar{l} I_{n}\right)^{-1} S_{k}^{-1}\left(A-\mu I_{n}\right)^{-1} \Pi_{k} .
$$

\section{Transformation of the Weyl functions and insertion of the eigenvalues}

Let now $m=2 p, H_{k} \geq 0(0<k \leq K)$, and put

$$
J=\left[\begin{array}{cc}
0 & I_{p} \\
I_{p} & 0
\end{array}\right] .
$$

Normalize the $m \times m$ fundamental solution $w$ of DCS by the initial condition

$$
w(0, l)=I_{m}
$$


Definition 3.1 41] The set $\mathcal{N}(w, K)$ of the Weyl functions $\varphi(l)$ for DCS (1.1) given on the interval $0<k \leq K$ is defined by the linear fractional transformation

$$
\varphi(l)=i\left[\begin{array}{ll}
I_{p} & 0
\end{array}\right] w(K, \bar{l})^{*} \chi(l)\left(\left[\begin{array}{ll}
0 & I_{p}
\end{array}\right] w(K, \bar{l})^{*} \chi(l)\right)^{-1} \quad\left(l \in \mathbb{C}_{+}\right),
$$

where $\mathbb{C}_{+}$is the open upper half-plane, and $\chi(l)$ are meromorphic in $\mathbb{C}_{+}$and non-degenerate $m \times p$ matrix functions with the $J$-property:

$$
\chi(l)^{*} \chi(l)>0, \quad \chi(l)^{*} J \chi(l) \geq 0 .
$$

Notice [4] that the inequality

$$
\mathcal{U}(l):=w(K, \bar{l})^{*} J w(K, \bar{l}) \geq J
$$

holds, and if $\operatorname{det}\left(\left[\begin{array}{ll}0 & I_{p}\end{array}\right] w(K, \bar{l})^{*} \chi(l)\right) \neq 0$, then $i\left(\varphi-\varphi^{*}\right) \leq 0$, i.e.,

$$
\widehat{\chi}(l)^{*} J \widehat{\chi}(l) \geq 0, \quad \widehat{\chi}(l):=\left[\begin{array}{c}
-i \varphi(l) \\
I_{p}
\end{array}\right] .
$$

If $H_{k} \geq 0$ for $0<k<\infty$ and $\mathcal{U}(l)>J$, then $\operatorname{det}\left(\left[\begin{array}{ll}0 & I_{p}\end{array}\right] w(K, \bar{l})^{*} \chi(l)\right) \neq 0$ for all $\chi$ satisfying (3.4), and it was shown in [41] that the Weyl functions $\varphi \in \cap_{K<\infty} \mathcal{N}(w, K)$ have the characteristic property:

$$
\sum_{k=0}^{\infty}\left[\begin{array}{ll}
I_{p} & i \varphi(l)^{*}
\end{array}\right] w(k, l)^{*} H_{k+1} w(k, l)\left[\begin{array}{c}
I_{p} \\
-i \varphi(l)
\end{array}\right]<\infty .
$$

Definition 3.2 The Weyl functions $\varphi(l)$ for DCS (1.1) $\left(H_{k} \geq 0\right)$ given on the semiaxis $0<k<\infty$ are defined by the condition (3.7).

In view of the representation (2.11) the normalized by the condition $\widetilde{w}(0, l)=$ $I_{m}$ solution $\widetilde{w}$ of the transformed system (2.8) is given by the equality

$$
\widetilde{w}(k, l)=v(k, l) w(k, l) v(0, l)^{-1} .
$$

The analog of Theorem 4 [35] on the Weyl functions of the transformed canonical system is true. 
Theorem 3.3 Suppose the Hamiltonian of the DCS (1.1), (1.2) on the interval $0<k \leq K$ is non-negative: $H_{k} \geq 0$, and the inequality in (3.5) is strict: $\mathcal{U}(l)>J$. If $S_{0}>0$, then the function $\widetilde{\varphi}$ of the form

$$
\widetilde{\varphi}(l)=i\left[\begin{array}{ll}
I_{p} & 0
\end{array}\right] J v(0, l) J \widehat{\chi}(l)\left(\left[\begin{array}{ll}
0 & I_{p}
\end{array}\right] J v(0, l) J \widehat{\chi}(l)\right)^{-1},
$$

where $\varphi \in \mathcal{N}(w, K)$ and $\hat{\chi}$ is given by (3.6), is a Weyl function of the transformed $D C S$ (2.8), i.e., $\widetilde{\varphi} \in \mathcal{N}(\widetilde{w}, K)$.

Pro of. Notice that $J v(0, l) J=\left(v(0, \bar{l})^{*}\right)^{-1}$. Hence according to (3.3), (3.8), and definition of $\widehat{\chi}$ in (3.6) we have

$$
\begin{aligned}
J v(0, l) J \widehat{\chi}(l)= & \left(v(0, \bar{l})^{*}\right)^{-1} w(K, \bar{l})^{*} v(K, \bar{l})^{*} \widetilde{\chi}(l)\left(\left[\begin{array}{ll}
0 & I_{p}
\end{array}\right] w(K, \bar{l})^{*} \chi(l)\right)^{-1} \\
& =\widetilde{w}(K, \bar{l})^{*} \widetilde{\chi}(l)\left(\left[\begin{array}{ll}
0 & I_{p}
\end{array}\right] w(K, \bar{l})^{*} \chi(l)\right)^{-1}
\end{aligned}
$$

where $\widetilde{\chi}(l):=\left(v(K, \bar{l})^{*}\right)^{-1} \chi(l)$. By Theorem 2.1 from $S_{0}>0$ we get $S_{k}>0$. Thus formulas (2.25) and (2.28) yield

$$
v(k, l)^{*} J v(k, l) \geq J, \quad v(k, \bar{l})^{*} J v(k, \bar{l}) \leq J \quad\left(l \in \mathbb{C}_{+}\right) .
$$

In particular, we have

$$
v(k, \bar{l})^{-1} J\left(v(k, \bar{l})^{*}\right)^{-1} \geq J \quad(k \geq 0) .
$$

Therefore if $\chi$ satisfies relations (3.4), then the matrix function $\tilde{\chi}(l)=$ $\left(v(K, \bar{l})^{*}\right)^{-1} \chi(l)$ satisfies relations (3.4) also. Moreover as $\mathcal{U}(l)>J$ we derive from (3.12) that

$$
\left(v(0, \bar{l})^{*}\right)^{-1} w(K, \bar{l})^{*} J w(K, \bar{l}) v(0, \bar{l})^{-1}>J .
$$

Taking into account formulas (3.4) and (3.13) we obtain

$$
\operatorname{det}\left(\left[\begin{array}{ll}
0 & I_{p}
\end{array}\right] \widetilde{w}(K, \bar{l})^{*} \widetilde{\chi}(l)\right)=\operatorname{det}\left(\left[\begin{array}{ll}
0 & I_{p}
\end{array}\right]\left(v(0, \bar{l})^{*}\right)^{-1} w(K, \bar{l})^{*} \chi(l)\right) \neq 0 .
$$

From (3.10), (3.12), and (3.14) follows the statement of the theorem.

The next theorem is closely related to Theorem 3.3. 
Theorem 3.4 Suppose $H_{k} \geq 0$ for $0<k<\infty, S_{0}>0$, and $\varphi(l)$ is a Weyl function for DCS (1.1) on the semiaxis $0<k<\infty$, i.e., matrix function $\varphi(l)$ satisfies [3.7). If the inequality $\operatorname{det}\left(\left[\begin{array}{ll}0 & I_{p}\end{array}\right] J v(0, l) J \widehat{\chi}(l)\right) \neq 0$ holds, then the matrix function $\widetilde{\varphi}(l)$ given by the equality (3.9) is a Weyl function of the transformed DCS (2.8) on the semiaxis:

$$
\sum_{k=0}^{\infty}\left[\begin{array}{ll}
I_{p} & i \widetilde{\varphi}(l)^{*}
\end{array}\right] \widetilde{w}(k, l)^{*} \widetilde{H}_{k+1} \widetilde{w}(k, l)\left[\begin{array}{c}
I_{p} \\
-i \widetilde{\varphi}(l)
\end{array}\right]<\infty .
$$

Here $\widetilde{w}$ is the solution of (2.8) normalized by the condition $\widetilde{w}(0, l)=I_{m}$.

Proof. From the equality

$$
\left(I_{m}+i l J H_{k+1}\right)^{*} J\left(I_{m}+i l J H_{k+1}\right)=J+i(l-\bar{l}) H_{k+1}
$$

and (1.1) it follows that

$$
w(k+1, l)^{*} J w(k+1, l)-w(k, l)^{*} J w(k, l)=i(l-\bar{l}) w(k, l)^{*} H_{k+1} w(k, l) .
$$

Hence one gets [4]

$$
\sum_{k=0}^{K-1} w(k, l)^{*} H_{k+1} w(k, l)=\frac{i}{l-\bar{l}}\left(J-w(K, l)^{*} J w(K, l)\right) .
$$

In view of (3.17) inequality (3.7) is equivalent to the boundedness of the forms

$$
\widehat{\chi}(l)^{*} w(K, l)^{*}(-J) w(K, l) \widehat{\chi}(l)<M(l) I_{m} \quad(K<\infty) .
$$

By the formulas (3.11) and (3.18) we have

$$
\widehat{\chi}(l)^{*} w(K, l)^{*} v(K, l)^{*}(-J) v(K, l) w(K, l) \widehat{\chi}(l)<M(l) I_{m} .
$$

Formula (3.9) yields the equality

$$
\left[\begin{array}{c}
-i \widetilde{\varphi}(l) \\
I_{p}
\end{array}\right]=J v(0, l) J \widehat{\chi}(l)\left(\left[\begin{array}{ll}
0 & I_{p}
\end{array}\right] J v(0, l) J \widehat{\chi}(l)\right)^{-1} .
$$

According to relations (3.8), (3.19) and (3.20) the inequalities

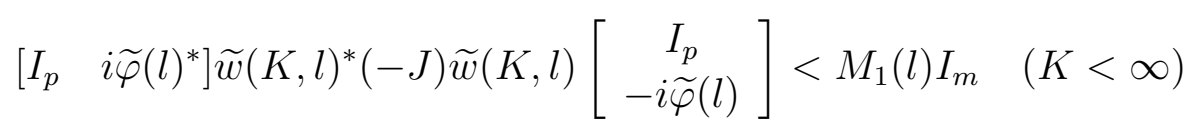


are true. Similar to the equivalence of (3.7) and (3.18) we get (3.15) from (3.21).

Insertion of the eigenvalues by the commutation methods have been studied in the important papers [11, 17, and useful references on the insertion of the eigenvalues into Jacobi operators one can find in 6, 44. Insertion of the eigenvalues and construction of the bound states using GBDT approach were investigated in the continuous case in [18, 35. The bound states and eigenvalues generated in DCS by the parameter matrix $A$ are described in the following theorem.

Theorem 3.5 Suppose $H_{k} \geq 0$ for $0<k<\infty, S_{0}>0$ and GBDT (2.8) is determined by the parameter matrices $A, S_{0}$, and $\Pi_{0}$. If $f \neq 0$ is an eigenvector of $A$, that is $A f=\mu f$, then the sequence $\left\{g_{k}\right\}$, where

$$
g_{k}=w_{0}(k)^{-1} J \Pi_{k}^{*} S_{k}^{-1} f,
$$

is a bound state of the DCS corresponding to the eigenvalue $\mu$, i.e., the relations

$$
g_{k}=\left(I_{m}+i \mu J \widetilde{H}_{k}\right) g_{k-1} \quad(k>0), \quad \sum_{k=0}^{\infty} g_{k}^{*} \widetilde{H}_{k+1} g_{k}<\infty
$$

are valid.

P r o of. First we shall prove the first relation in (3.23). By (1.2), (2.2), and (2.14) we get

$$
\begin{gathered}
J \Pi_{k}^{*} S_{k}^{-1} f= \\
=\left(I_{m}+i \mu J H_{k}+J H_{k} J \Pi_{k-1}^{*} S_{k-1}^{-1} \Pi_{k-1}-J \Pi_{k}^{*} S_{k}^{-1} \Pi_{k} J H_{k}\right) J \Pi_{k-1}^{*} S_{k-1}^{-1} f .
\end{gathered}
$$

According to (2.21), (3.22), and (3.24) the first relation in (3.23) is true.

To prove the second relation in (3.23) for $\mu \neq \bar{\mu}$ analogously to (3.17) we get

$$
\sum_{k=0}^{K-1} \widetilde{w}(k, l)^{*} \widetilde{H}_{k+1} \widetilde{w}(k, l)=\frac{i}{l-\bar{l}}\left(J-\widetilde{w}(K, l)^{*} J \widetilde{w}(K, l)\right) .
$$


From the first relation in (3.23) it follows that

$$
g_{k}=\widetilde{w}(k, \mu) g_{0} .
$$

Therefore, if $\mu \neq \bar{\mu}$, then in view of (3.25) and (3.26) we obtain

$$
\sum_{k=0}^{K-1} g_{k}^{*} \widetilde{H}_{k+1} g_{k}=\frac{i}{\mu-\bar{\mu}}\left(g_{0}^{*} J g_{0}-g_{K}^{*} J g_{K}\right) \text {. }
$$

Finally notice that formulas (2.4), (2.25), and (3.22) yield:

$$
g_{k}^{*} J g_{k}=-i f^{*}\left(S_{k}^{-1} A-A^{*} S_{k}^{-1}\right) f=-i(\mu-\bar{\mu}) f^{*} S_{k}^{-1} f .
$$

According to Theorem 2.1 the inequalities $S_{k}>0$ hold. Hence from (3.27) and (3.28) it follows that

$$
\sum_{k=0}^{\infty} g_{k}^{*} \widetilde{H}_{k+1} g_{k} \leq f^{*} S_{0}^{-1} f
$$

and the second relation in (3.23) is valid. The proof of (3.29) in the general case that includes real eigenvalues of $A$ is somewhat more complicated. For that purpose we shall consider $g_{k}^{*} \widetilde{H}_{k+1} g_{k}$. From (2.22), (2.25), and (3.22) we derive

$$
g_{k}^{*} \widetilde{H}_{k+1} g_{k}=f^{*} S_{k}^{-1} \Pi_{k} J\left(w_{0}(k+1) w_{0}(k)^{-1}\right)^{*} H_{k+1} J \Pi_{k}^{*} S_{k}^{-1} f .
$$

Thus taking into account (1.2) and (2.7) we get

$$
\begin{gathered}
g_{k}^{*} \widetilde{H}_{k+1} g_{k}= \\
=f^{*} S_{k}^{-1}\left(\Pi_{k} J H_{k+1} J \Pi_{k}^{*}-\Pi_{k} J H_{k+1} J \Pi_{k+1}^{*} S_{k+1}^{-1} \Pi_{k+1} J H_{k+1} J \Pi_{k}^{*}\right) S_{k}^{-1} f .
\end{gathered}
$$

Using (1.2) and (2.2) rewrite (3.31) as

$$
g_{k}^{*} \widetilde{H}_{k+1} g_{k}=f^{*} S_{k}^{-1}\left(\Pi_{k} J H_{k+1} J \Pi_{k}^{*}-\Pi_{k} J H_{k+1} J \Pi_{k}^{*} S_{k+1}^{-1} \Pi_{k} J H_{k+1} J \Pi_{k}^{*}\right) S_{k}^{-1} f .
$$

Therefore in view of (2.3) we obtain

$$
\begin{aligned}
g_{k}^{*} \widetilde{H}_{k+1} g_{k}=f^{*} S_{k}^{-1}\left(S_{k+1}\right. & \left.-S_{k}-\left(S_{k+1}-S_{k}\right) S_{k+1}^{-1}\left(S_{k+1}-S_{k}\right)\right) S_{k}^{-1} f= \\
& =f^{*}\left(S_{k}^{-1}-S_{k+1}^{-1}\right) f .
\end{aligned}
$$

By (3.33) inequality (3.29) is true for $\mu \in \mathbb{R}$ also. 


\section{Matrix Toda lattice}

In this section we shall consider the MTL generalization (1.3), (1.4) of the well known Toda lattice. For this purpose we shall need an equivalent representation of (1.3) in the zero curvature form [41]

$$
\frac{d}{d t} G(k, t, l)+G(k, t, l) F(k-1, t, l)-F(k, t, l) G(k, t, l)=0,
$$

where

$$
\begin{gathered}
G(k, t, l):=-l \mathcal{P}_{1}+i \xi(k, t), \quad F(k, t, l):=-l \mathcal{P}_{2}-i \psi(k, t), \\
\mathcal{P}_{1}:=\left[\begin{array}{ll}
I_{p} & 0 \\
0 & 0
\end{array}\right], \quad \mathcal{P}_{2}:=\left[\begin{array}{cc}
0 & 0 \\
0 & I_{p}
\end{array}\right], \quad \mathcal{P}_{1} \mathcal{P}_{2}=\mathcal{P}_{2} \mathcal{P}_{1}=0, \\
\xi(k, t):=\left[\begin{array}{rr}
-i Q(k, t) & C(k, t) \\
C(k, t)^{-1} & 0
\end{array}\right], \quad \psi(k, t):=\left[\begin{array}{lr}
0 & C(k+1, t) \\
C(k, t)^{-1} & 0
\end{array}\right] .
\end{gathered}
$$

See [2, 9, 14, 15, 26] for the references on the lattice models and [1] for the discrete version of the zero curvature equation.

To describe a Bäcklund-Darboux transformation for the MTL introduce Hamiltonians $\left\{H_{k}(t)\right\}$ by the equalities

$$
H_{k}(t):=J U(k, t) \eta(k, t) U(k, t)^{*} J, \quad \eta(k, t):=\left[\begin{array}{ll}
C(k, t) & 0 \\
0 & 0
\end{array}\right],
$$

where $J$ is given by the equality (3.1),

$$
U(k, t)=U(k-1, t) \xi(k, t)^{-1}, \quad U(0, t) J U(0, t)^{*}=J .
$$

Notice that according to formulas (1.4), (4.4) and (4.6) we have

$$
\xi(k, t) J \xi(k, t)^{*}=J, \quad U(k, t) J U(k, t)^{*}=J .
$$

By (4.5) and the second relation in (4.7) $H_{k}$ satisfies condition (1.2).

Add now a new variable $t$ in the notations of Section 2. Similar to Section 2 , if given a solution $\{C(k, t), Q(k, t)\}$ of (1.3), (1.4), we choose $n>0$ and 
three parameter matrices $A, S_{0}(0)$, and $\widehat{\Pi}_{0}(0)$ that satisfy the matrix identity

$$
A S_{0}(0)-S_{0}(0) A^{*}=i \widehat{\Pi}_{0}(0) J \widehat{\Pi}_{0}(0)^{*} \quad\left(S_{0}(0)=S_{0}(0)^{*}\right)
$$

to construct a new solution. We put

$$
\widehat{\Pi}_{k}(t)=\Pi_{k}(t) U(k, t)
$$

and so according to (4.7) identity (4.8) is equivalent to the identity $A S_{0}(0)-$ $S_{0}(0) A^{*}=i \Pi_{0}(0) J \Pi_{0}(0)^{*}$. In view of (4.6), (4.7) and (4.9) formulas (2.2) are equivalent to the equalities

$$
\widehat{\Pi}_{k}(t)=\widehat{\Pi}_{k-1}(t) \xi(k, t)^{-1}-i A \widehat{\Pi}_{k-1}(t) \mathcal{P}_{2} .
$$

Thus $\left\{\widehat{\Pi}_{k}(t)\right\}$ is uniquely defined via (4.10) by the initial value $\widehat{\Pi}_{0}(0)$ and equation

$$
\frac{d}{d t} \widehat{\Pi}_{0}(t)=A \widehat{\Pi}_{0}(t) \mathcal{P}_{2}+i \widehat{\Pi}_{0}(t) \psi(0, t) .
$$

Using (4.5), (4.7) and (4.9) rewrite now equation (2.3) in the form

$$
S_{k}(t)=S_{k-1}(t)+\widehat{\Pi}_{k-1}(t) \zeta(k, t) \widehat{\Pi}_{k-1}(t)^{*},
$$

where $\zeta(k, t)=\xi(k, t)^{-1} \eta(k, t)\left(\xi(k, t)^{*}\right)^{-1}$. By (4.4) we have

$$
\xi(l, t)^{-1}=\left[\begin{array}{lr}
0 & C(l, t) \\
C(l, t)^{-1} & i C(l, t)^{-1} Q(l, t) C(l, t)
\end{array}\right],
$$

For the sake of brevity we shall sometimes omit $t$ in our notations. In view of (1.3), (1.4), and (4.13) it is immediate that

$$
\zeta(k)=\left[\begin{array}{rr}
0 & 0 \\
0 & C(k)^{-1}
\end{array}\right], \quad \frac{d}{d t} \zeta(k)=-\frac{1}{2}\left[\begin{array}{lr}
0 & 0 \\
0 & C(k)^{-1} Q(k)+Q(k)^{*} C(k)^{-1}
\end{array}\right] .
$$

We define $\left\{S_{k}(t)\right\}$ by the formulas (4.12), initial value $S_{0}(0)$, and equation

$$
\frac{d}{d t} S_{0}(t)=\frac{1}{2}\left(A S_{0}(t)+S_{0}(t) A^{*}+i \widehat{\Pi}_{0}(t)\left[\begin{array}{rr}
0 & -I_{p} \\
I_{p} & 0
\end{array}\right] \widehat{\Pi}_{0}(t)^{*}\right) .
$$

Then the identity

$$
A S_{0}(t)-S_{0}(t) A^{*}=i \widehat{\Pi}_{0}(t) J \widehat{\Pi}_{0}(t)^{*}=i \Pi_{0}(t) J \Pi_{0}(t)^{*} .
$$


is valid. Indeed, by formulas (4.8), (4.11), and (4.15) for $Z(t)=A S_{0}(t)-$ $S_{0}(t) A^{*}-i \widehat{\Pi}_{0}(t) J \widehat{\Pi}_{0}(t)^{*}$ we have $Z^{\prime}=\frac{1}{2}\left(A Z+Z A^{*}\right)$ and $Z(0)=0$. Hence the identity $Z(t) \equiv 0$ is valid, i.e., (4.16) is true. By the considerations of Section 2 formula (4.16) yields

$$
A S_{k}(t)-S_{k}(t) A^{*}=i \Pi_{k}(t) J \Pi_{k}(t)^{*}
$$

Formulas (4.10)-(4.12), (4.15) introduce $\widehat{\Pi}_{k}$ and $S_{k}$ independently of the considerations of Section 1, relations (4.5), (4.6), and (4.9) granting transfer to the auxiliary discrete canonical systems.

Now a Bäcklund-Darboux transformation for MTL is given by the relations

$$
\begin{gathered}
\widetilde{C}(k, t)=C(k, t)+X_{22}(k-1, t) \quad(k>0), \quad \widetilde{C}(0, t)=\left(C(0, t)-X_{11}(0, t)\right)^{-1}, \\
\widetilde{Q}(k, t)=Q(k, t)+i\left(X_{21}(k-1, t)-X_{21}(k, t)\right) \quad(k>0),
\end{gathered}
$$

where $X_{i j}(k, t)$ are $p \times p$ blocks of the matrix

$$
X(k, t)=\left[\begin{array}{cc}
X_{11}(k, t) & X_{12}(k, t) \\
X_{21}(k, t) & X_{22}(k, t)
\end{array}\right]=\widehat{\Pi}_{k}(t)^{*} S_{k}(t)^{-1} \widehat{\Pi}_{k}(t) .
$$

Theorem 4.1 Suppose matrix functions $C(k, t)$ and $Q(k, t)$ satisfy $M T L$ (1.3), (1.4) and $C(0, t)$ is continuous. Suppose additionally that matrix functions $C(0, t)^{-1}-X_{11}(0, t)$ and matrix functions $S_{k}(t)$ given by $\sqrt{4.12)}$ and (4.15) are invertible in the domain $-\varepsilon_{1}<t<\varepsilon_{2}$, $0 \leq k<K$. Then the matrix functions $\widetilde{C}$ and $\widetilde{Q}$ are well defined by (4.18)-(4.20) and satisfy $M T L$ in the domain $-\varepsilon_{1}<t<\varepsilon_{2}, 0<k<K$.

Proof. To prove the theorem we shall obtain derivatives in $t$ for $\widehat{\Pi}_{k}, S_{k}$, $\widehat{\Pi}_{k} S_{k}^{-1}$, and, finally, $\widehat{w}_{A}(k, l):=U(k)^{-1} w_{A}(k, l) U(k)$. First let us recall (4.11) and show by induction that

$$
\frac{d}{d t} \widehat{\Pi}_{k}(t)=A \widehat{\Pi}_{k}(t) \mathcal{P}_{2}+i \widehat{\Pi}_{k}(t) \psi(k, t)
$$


In view of (4.10) we get

$$
\widehat{\Pi}_{l}^{\prime}=\widehat{\Pi}_{l-1}^{\prime} \xi(l)^{-1}-i A \widehat{\Pi}_{l-1}^{\prime} \mathcal{P}_{2}-\widehat{\Pi}_{l-1} \xi(l)^{-1} \xi(l)^{\prime} \xi(l)^{-1} .
$$

Taking into account (1.3) we see that

$$
\xi(l)^{\prime}=\left[\begin{array}{lr}
-i\left(C(l+1) C(l)^{-1}-C(l) C(l-1)^{-1}\right) & Q(l) C(l) \\
-C(l)^{-1} Q(l) & 0
\end{array}\right] .
$$

Suppose that (4.21) is true for $k=l-1$. Then the definition of $\psi$ and equalities (4.13), (4.22) yield

$$
\begin{aligned}
\widehat{\Pi}_{l}^{\prime}= & \left(A \widehat{\Pi}_{l-1} \mathcal{P}_{2}+i \widehat{\Pi}_{l-1} \psi(l-1)\right) \xi(l)^{-1}-i A\left(A \widehat{\Pi}_{l-1} \mathcal{P}_{2}+i \widehat{\Pi}_{l-1} \psi(l-1)\right) \mathcal{P}_{2} \\
& -\widehat{\Pi}_{l-1} \xi(l)^{-1} \xi(l)^{\prime} \xi(l)^{-1}=A\left(-i A \widehat{\Pi}_{l-1} \mathcal{P}_{2}+\widehat{\Pi}_{l-1} \xi(l)^{-1} \mathcal{P}_{2}\right) \\
+ & A \widehat{\Pi}_{l-1} \xi(l)^{-1} \mathcal{P}_{1}+\widehat{\Pi}_{l-1} \xi(l)^{-1}\left(i \xi(l) \psi(l-1)-\xi(l)^{\prime}\right) \xi(l)^{-1}
\end{aligned}
$$

Using (4.13) and (4.23) we calculate directly that

$$
\xi(l)^{-1} \mathcal{P}_{1}=\mathcal{P}_{2} \psi(l), \quad\left(i \xi(l) \psi(l-1)-\xi(l)^{\prime}\right) \xi(l)^{-1}=i \psi(l) .
$$

From (4.10), (4.24) and (4.25) follows equation (4.21) for $k=l$, i.e., (4.21) holds for all $k \geq 0$.

Taking into account (4.15) and (4.21) one can prove that

$$
\frac{d}{d t} S_{k}(t)=\frac{1}{2}\left(A S_{k}(t)+S_{k}(t) A^{*}+i \widehat{\Pi}_{k}(t)\left[\begin{array}{rr}
0 & -I_{p} \\
I_{p} & 0
\end{array}\right] \widehat{\Pi}_{k}(t)^{*}\right) .
$$

Suppose (4.26) is true for $k=l-1$. Then from formulas (4.12) and (4.21) and equation (4.26) for $k=l-1$ we obtain

$$
\begin{gathered}
S_{l}^{\prime}=\frac{1}{2}\left(A S_{l-1}+S_{l-1} A^{*}+i \widehat{\Pi}_{l-1}\left[\begin{array}{rr}
0 & -I_{p} \\
I_{p} & 0
\end{array}\right] \widehat{\Pi}_{l-1}^{*}\right)+\widehat{\Pi}_{l-1} \zeta^{\prime}(l) \widehat{\Pi}_{l-1}^{*} \\
+\left(A \widehat{\Pi}_{l-1} \mathcal{P}_{2}+i \widehat{\Pi}_{l-1} \psi(l-1)\right) \zeta(l) \widehat{\Pi}_{l-1}^{*}+\widehat{\Pi}_{l-1} \zeta(l)\left(A \widehat{\Pi}_{l-1} \mathcal{P}_{2}+i \widehat{\Pi}_{l-1} \psi(l-1)\right)^{*} .
\end{gathered}
$$


By (4.10), (4.12), and (4.14) after some calculations we derive now (4.26) for $k=l$ and thus for all $k \geq 0$.

By (4.21) and (4.26) one can show that

$$
\begin{aligned}
\frac{d}{d t}\left(\widehat{\Pi}_{k}(t)^{*} S_{k}(t)^{-1}\right) & =-\mathcal{P}_{1} \widehat{\Pi}_{k}(t)^{*} S_{k}(t)^{-1} A-i J \widetilde{\psi}(k, t) J \widehat{\Pi}_{k}(t)^{*} S_{k}(t)^{-1}, \\
\widetilde{\psi}(k, t) & :=\left[\begin{array}{lr}
0 & \widetilde{C}(k+1, t) \\
\widetilde{C}(k, t)^{-1} & 0
\end{array}\right]
\end{aligned}
$$

where matrix functions $\widetilde{C}(k, t)$ are given by (4.18), (4.20). Indeed, by (4.21) and (4.26) we have

$$
\begin{gathered}
\left(\widehat{\Pi}_{k}^{*} S_{k}^{-1}\right)^{\prime}=\mathcal{P}_{2} \widehat{\Pi}_{k}^{*} A^{*} S_{k}^{-1}-i J \psi(k) J \widehat{\Pi}_{k}^{*} S_{k}^{-1} \\
-\frac{1}{2} \widehat{\Pi}_{k}^{*} S_{k}^{-1}\left(A S_{k}+S_{k} A^{*}+i \widehat{\Pi}_{k}\left[\begin{array}{rr}
0 & -I_{p} \\
I_{p} & 0
\end{array}\right] \widehat{\Pi}_{k}^{*}\right) S_{k}^{-1}=-\frac{1}{2} \widehat{\Pi}_{k}^{*} S_{k}^{-1} A \\
-\frac{1}{2}\left(\mathcal{P}_{1}-\mathcal{P}_{2}\right) \widehat{\Pi}_{k}^{*} A^{*} S_{k}^{-1}-i J \psi(k) J \widehat{\Pi}_{k}^{*} S_{k}^{-1}+\frac{i}{2} X(k)\left(\mathcal{P}_{1}-\mathcal{P}_{2}\right) J \widehat{\Pi}_{k}^{*} S_{k}^{-1} .
\end{gathered}
$$

Rewrite (4.17) in the form

$$
A^{*} S_{k}^{-1}=S_{k}^{-1} A-i S_{k}^{-1} \widehat{\Pi}_{k} J \widehat{\Pi}_{k}^{*} S_{k}^{-1} .
$$

Substitute $A^{*} S_{k}^{-1}$ by the right-hand side of (4.31) in the right-hand side of (4.30) to get

$$
\begin{gathered}
\frac{d}{d t}\left(\widehat{\Pi}_{k}(t)^{*} S_{k}(t)^{-1}\right)=-\mathcal{P}_{1} \widehat{\Pi}_{k}(t)^{*} S_{k}(t)^{-1} A \\
-i\left[\begin{array}{lr}
0 & C(k, t)^{-1}-X_{11}(k, t) \\
C(k+1, t)+X_{22}(k, t) & 0
\end{array}\right] \widehat{\Pi}_{k}(t)^{*} S_{k}(t)^{-1} .
\end{gathered}
$$

So according to (4.18) it remains to obtain the equality

$$
\widetilde{C}(k, t)^{-1}=C(k, t)^{-1}-X_{11}(k, t)
$$

to prove (4.28). By definition (4.18) equality (4.32) is true for $k=0$. To prove (4.32) for $k>0$ notice that $\eta(k) J \xi(k)=\mathcal{P}_{1}$ and in view of (4.5)-(4.7) derive the relation ( 41$]$, formula (8.2.7)):

$$
U(k, t) \mathcal{P}_{1}=J H_{k}(t) U(k-1, t) .
$$


Putting

$$
\widetilde{\xi}(k, t):=\widetilde{U}(k, t)^{-1} \widetilde{U}(k-1, t), \quad \widetilde{U}(k, t):=w_{0}(k, t)^{-1} U(k, t),
$$

consider the expression $\widetilde{\xi}$. By (2.7) we have

$$
\begin{gathered}
\widetilde{\xi}(k)=U(k)^{-1} w_{0}(k) w_{0}(k-1)^{-1} U(k-1)= \\
U(k)^{-1}\left(I_{m}-J \Pi_{k}^{*} S_{k}^{-1} \Pi_{k} J H_{k}+J H_{k} J \Pi_{k-1}^{*} S_{k-1}^{-1} \Pi_{k-1}\right) U(k-1) .
\end{gathered}
$$

Hence taking into account formulas (4.7), (4.33) and definitions (4.6), (4.9) and (4.20) we get

$$
\begin{gathered}
\widetilde{\xi}(k)=\xi(k)-J X(k) \mathcal{P}_{1}+\mathcal{P}_{1} J X(k-1)= \\
{\left[\begin{array}{lr}
-i\left(Q(k)+i\left(X_{21}(k-1)-X_{21}(k)\right)\right) & C(k)+X_{22}(k-1) \\
C(k)^{-1}-X_{11}(k) & 0
\end{array}\right] .}
\end{gathered}
$$

By (4.36) the left lower $p \times p$ block $\left(\widetilde{\xi}(k) J \widetilde{\xi}(k)^{*}\right)_{21}$ of $\widetilde{\xi}(k) J \widetilde{\xi}(k)^{*}$ equals $\left(C(k)^{-1}-X_{11}(k)\right)\left(C(k)+X_{22}(k-1)\right)$, and by (4.34) $\widetilde{\xi}$ is $J$-unitary. Thus we have

$$
\left(C(k)^{-1}-X_{11}(k)\right)\left(C(k)+X_{22}(k-1)\right)=I_{p},
$$

i.e., equality (4.32) holds. Moreover, from the definitions (4.18), (4.19) and equalities (4.32) and (4.36) it follows that

$$
\widetilde{\xi}(k, t)=\left[\begin{array}{lr}
-i \widetilde{Q}(k, t) & \widetilde{C}(k) \\
\widetilde{C}(k)^{-1} & 0
\end{array}\right] .
$$

Now again use the fact that $\xi$ is $J$-unitary to show that (1.4) for $\widetilde{C}$ and $\widetilde{Q}$ holds: $C(k) Q(k)^{*}=Q(k) C(k)$.

Next we shall calculate the derivative of

$$
\widehat{w}_{A}(k, t, l)=U(k, t)^{-1} w_{A}(k, t, l) U(k, t) .
$$


According to the definitions (2.6) and (4.9) the function $\widehat{w}_{A}$ has the form

$$
\widehat{w}_{A}(k, t, l)=I_{m}-i J \widehat{\Pi}_{k}(t)^{*} S_{k}(t)^{-1}\left(A-l I_{n}\right)^{-1} \widehat{\Pi}_{k}(t) .
$$

Using formulas (4.21) and (4.28) and equality $A=\left(A-l I_{n}\right)+l I_{n}$ we obtain easily:

$$
\begin{array}{r}
\frac{d}{d t} \widehat{w}_{A}(k, l)=l\left(-J \mathcal{P}_{1} J\left(\widehat{w}_{A}(k, l)-I_{m}\right)+\left(\widehat{w}_{A}(k, l)-I_{m}\right) \mathcal{P}_{2}\right)- \\
i \widetilde{\psi}(k)\left(\widehat{w}_{A}(k, l)-I_{m}\right)+i\left(\widehat{w}_{A}(k, l)-I_{m}\right) \psi(k)+i J \mathcal{P}_{1} X(k)-i J X(k) \mathcal{P}_{2} .
\end{array}
$$

Now in view of (4.18), (4.29) and (4.32) after evident transformations of (4.40) we get the result

$$
\frac{d}{d t} \widehat{w}_{A}(k, t, l)=\widetilde{F}(k, t, l) \widehat{w}_{A}(k, t, l)-\widehat{w}_{A}(k, t, l) F(k, t, l),
$$

where

$$
F(k, t, l)=-l \mathcal{P}_{2}-i \psi(k, t), \quad \widetilde{F}(k, t, l)=-l \mathcal{P}_{2}-i \widetilde{\psi}(k, t)
$$

We shall need also a reformulation of (2.15) that follows from the relations (4.33), (4.35) and (4.38):

$$
\widehat{w}_{A}(k, t, l) G(k, t, l)=\widetilde{G}(k, t, l) \widehat{w}_{A}(k-1, t, l),
$$

where

$$
G(k, t, l)=-l \mathcal{P}_{1}+i \xi(k, t), \quad \widetilde{G}(k, t, l)=-l \mathcal{P}_{1}+i \widetilde{\xi}(k, t)
$$

Recall that system (1.3) is equivalent to the zero curvature equation (4.1). Equation (4.1) is a compatibility condition for the systems

$$
W(k, t, l)=G(k, t, l) W(k-1, t, l), \quad \frac{d}{d t} W(k, t, l)=F(k, t, l) W(k, t, l) .
$$


Thus by (1.3) there exists an $m \times m$ matrix function $W(\operatorname{det} W \neq 0)$ that satisfies (4.45) [41]. For $\widetilde{W}:=\widehat{w}_{A} W$ formula (4.41) and the second relation in (4.45) now yield:

$$
\frac{d}{d t} \widetilde{W}(k, t, l)=\widetilde{F}(k, t, l) \widetilde{W}(k, t, l) .
$$

Formula (4.43) and the first relation in (4.45) yield

$$
\widetilde{W}(k, t, l)=\widehat{w}_{A}(k, t, l) G(k, t, l) W(k-1, t, l)=\widetilde{G}(k, t, l) \widetilde{W}(k-1, t, l) .
$$

In view of formula (4.37) and the second relations in formulas (4.42) and (4.44) the compatibility condition

$$
\frac{d}{d t} \widetilde{G}(k, t, l)+\widetilde{G}(k, t, l) \widetilde{F}(k-1, t, l)-\widetilde{F}(k, t, l) \widetilde{G}(k, t, l)=0
$$

for systems (4.46) and (4.47) is equivalent to the system

$$
\left\{\begin{array}{c}
\widetilde{Q}^{\prime}(k, t)=\widetilde{C}(k+1, t) \widetilde{C}(k, t)^{-1}-\widetilde{C}(k, t) \widetilde{C}(k-1, t)^{-1} \\
\widetilde{C}^{\prime}(k, t)=\widetilde{Q}(k, t) \widetilde{C}(k, t) .
\end{array}\right.
$$

Thus $\widetilde{Q}$ and $\widetilde{C}$ satisfy MTL.

\section{Explicit solutions}

In this section we shall consider first the case of the trivial MTL (1.3), (1.4) solution that is given by the functions

$$
C(k, t) \equiv I_{p} \quad(k \geq 0), \quad Q(k, t) \equiv 0 \quad(k>0) .
$$

Starting from these initial $C$ and $Q$ we shall use GBDT to get explicit formulas for a wide class of the MTL solutions, fundamental DCS solutions and evolution of the Weyl functions. According to (4.4) we have

$$
\psi(k, t) \equiv J \quad(k \geq 0), \quad \xi(k, t) \equiv J \quad(k>0) .
$$

Putting $U(0, t) \equiv I_{m}$ we derive also

$$
U(k, t)=J^{k}, \quad H_{k}(t)=J^{k} \mathcal{P}_{2} J^{k} .
$$


Thus the normalized fundamental solution of the DCS has the form

$$
w(k, l)=\prod_{l=1}^{k}\left(I_{m}+i l J^{l+1} \mathcal{P}_{2} J^{l}\right)
$$

Notice that $\mathcal{P}_{1}+\mathcal{P}_{2}=I_{m}$ and $J \mathcal{P}_{2} J=\mathcal{P}_{1}$. Hence we obtain

$$
\left(I_{m}+i l J \mathcal{P}_{2}\right)\left(I_{m}+i l J \mathcal{P}_{1}\right)=I_{m}+i l J-l^{2} \mathcal{P}_{1}=\left[\begin{array}{lr}
\left(1-l^{2}\right) I_{p} & i l I_{p} \\
i l I_{p} & I_{p}
\end{array}\right] .
$$

From (5.5) follows representation

$$
\left(I_{m}+i l J \mathcal{P}_{2}\right)\left(I_{m}+i l J \mathcal{P}_{1}\right)=T D_{l} T^{-1},
$$

where

$$
\begin{gathered}
T=\left[\begin{array}{lr}
I_{p} & I_{p} \\
\frac{l+\sqrt{l^{2}-4}}{2 i} I_{p} & \frac{l-\sqrt{l^{2}-4}}{2 i} I_{p}
\end{array}\right], \quad D_{l}=\left[\begin{array}{cc}
z_{1} I_{p} & 0 \\
0 & z_{2} I_{p}
\end{array}\right], \\
z_{1,2}=\frac{1}{2}\left(2-l^{2} \pm l \sqrt{l^{2}-4}\right) .
\end{gathered}
$$

Later we shall choose $\sqrt{l^{2}-4} \in \mathbb{C}_{+}$for $l \in \mathbb{C}_{+}$. As $J^{2}=I_{m}$, using (5.4) and (5.6) we obtain:

$w(k, l)=T D_{l}^{l} T^{-1}$ for $k=2 l, \quad w(k, l)=\left(I_{m}+i l J \mathcal{P}_{1}\right) T D_{l}^{l} T^{-1}$ for $k=2 l+1$.

We shall apply now Theorem 4.1 to construct explicit MTL solutions.

Theorem 5.1 Fix $n>0$, two $n \times n$ parameter matrices $A$ and $S_{0}(0)$ and $n \times m$ parameter matrix $\widehat{\Pi}_{0}(0)=\left[\theta_{1}(0,0) \quad \theta_{2}(0,0)\right]$ with $n \times p$ blocks $\theta_{l}(0,0)$, such that

$$
\begin{gathered}
A=\alpha+\alpha^{-1}, \quad \operatorname{det}\left(\alpha-\alpha^{-1}\right) \neq 0, \quad S_{0}(0)>0, \\
\operatorname{det}\left(I_{p}-\left[\begin{array}{ll}
I_{p} & 0
\end{array}\right] \widehat{\Pi}_{0}(0)^{*} S_{0}(0)^{-1} \widehat{\Pi}_{0}(0)\left[\begin{array}{c}
I_{p} \\
0
\end{array}\right]\right) \neq 0,
\end{gathered}
$$

and (4.8) holds. Put now

$$
\widetilde{C}(k, t)=I_{p}+X_{22}(k-1, t) \quad(k>0), \quad \widetilde{C}(0, t)=\left(I_{p}-X_{11}(0, t)\right)^{-1}
$$




$$
\widetilde{Q}(k, t)=i\left(X_{21}(k-1, t)-X_{21}(k, t)\right) \quad(k>0),
$$

where $X_{i j}(k, t)$ are $p \times p$ blocks of the matrix

$$
X(k, t)=\left[\begin{array}{ll}
X_{11}(k, t) & X_{12}(k, t) \\
X_{21}(k, t) & X_{22}(k, t)
\end{array}\right]=\widehat{\Pi}_{k}(t)^{*} S_{k}(t)^{-1} \widehat{\Pi}_{k}(t) .
$$

Define matrices $\widehat{\Pi}_{k}$ and $S_{k}$ in the right-hand side of [5.14) by the equalities

$$
\begin{aligned}
& {\left[\begin{array}{l}
\theta_{+} \\
\theta_{-}
\end{array}\right]=\left(\left[\begin{array}{cc}
i \alpha^{-1} & i \alpha \\
I_{p} & I_{p}
\end{array}\right]\right)^{-1}\left[\begin{array}{l}
\theta_{1}(0,0) \\
\theta_{2}(0,0)
\end{array}\right]} \\
& \widehat{\Pi}_{k}(t)=\left[\theta_{1}(k, t) \quad \theta_{2}(k, t)\right], \quad \theta_{1}(k, t)=(-i \alpha)^{k-1} e^{\alpha t} \theta_{+}+\left(-i \alpha^{-1}\right)^{k-1} e^{\alpha^{-1} t} \theta_{-}, \\
& \theta_{2}(k, t)=(-i \alpha)^{k} e^{\alpha t} \theta_{+}+\left(-i \alpha^{-1}\right)^{k} e^{\alpha^{-1} t} \theta_{-}, \\
& S_{k}(t)=S_{0}(t)+\sum_{l=0}^{k-1} \theta_{2}(l, t) \theta_{2}(l, t)^{*}, \quad S_{0}(t)=\frac{1}{2} \theta_{1}(0, t) \theta_{1}(0, t)^{*}+e^{\frac{1}{2} A t} q(t) e^{\frac{1}{2} A^{*} t}, \\
& q(t)=S_{0}(0)-\frac{1}{2} \theta_{1}(0,0) \theta_{1}(0,0)^{*}+ \\
& \frac{1}{4} \int_{0}^{t} e^{-\frac{1}{2} A u}\left(A \theta_{1}(0, u) \theta_{1}(0, u)^{*}+\theta_{1}(0, u) \theta_{1}(0, u)^{*} A^{*}\right) e^{-\frac{1}{2} A^{*} u} d u .
\end{aligned}
$$

Then for some $\varepsilon_{1}>0, \varepsilon_{2}>0$ matrix functions $\widetilde{C}$ and $\widetilde{Q}$ are well defined by (5.12)-(5.18) and satisfy MTL in the domain $-\varepsilon_{1}<t<\varepsilon_{2}, 0<k<\infty$.

Pr o of. One can check easily that according to the second relation in (5.10) the square matrix in the right-hand side of (5.15) is invertible. By (5.15) the right-hand sides in the second and third relations in (5.16) at $k=0, t=0$ turn into $\theta_{1}(0,0)$ and $\theta_{2}(0,0)$, respectively, i.e., $\widehat{\Pi}$ is well defined by (5.16). Moreover, from (5.16) we get $\theta_{1}^{\prime}(0, t)=i \theta_{2}(0, t)$ and $\theta_{2}^{\prime}(0, t)=A \theta_{2}(0, t)+$ $i \theta_{1}(0, t)$. Hence in view of (5.2) $\widehat{\Pi}_{0}(t)$ satisfies (4.11). Notice also that

$$
\left(\theta_{1}(0, t) \theta_{1}(0, t)^{*}\right)^{\prime}=i\left(\theta_{2}(0, t) \theta_{1}(0, t)^{*}-\theta_{1}(0, t) \theta_{2}(0, t)^{*}\right) .
$$


From the equalities $\theta_{1}(k, t)=\theta_{2}(k-1, t)$ and $\theta_{2}(k, t)=\theta_{1}(k-1, t)-i A \theta_{2}(k-$ $1, t)$ it follows that $\widehat{\Pi}_{k}(t)$ satisfies (4.10). According to (5.17) we see that $S_{k}(t)$ satisfies (4.12). Finally formulas (5.17), (5.18) yield

$$
\left(S_{0}(t)-\frac{1}{2} \theta_{1}(0, t) \theta_{1}(0, t)^{*}\right)^{\prime}=\frac{1}{2}\left(A S_{0}(t)+S_{0}(t) A^{*}\right),
$$

and taking into account (5.19) we obtain (4.15). Therefore matrices $\widetilde{C}$ and $\widetilde{Q}$ coincide with the matrices generated in Theorem 4.1, when $C$ and $Q$ are given by (5.1). As $S_{0}(0)>0$ and inequality (5.11) holds, so for some $\varepsilon_{1}>0$, $\varepsilon_{2}>0$ we have $S_{0}(t)>0, \operatorname{det}\left(I_{p}-X_{11}(0, t)\right) \neq 0$ on the interval $-\varepsilon_{1}<t<\varepsilon_{2}$. Taking into account the first equality in (5.17) we derive $S_{k}(t)>0$ also. The conditions of Theorem 4.1 are fulfilled, i.e., $\widetilde{C}$ and $\widetilde{Q}$ satisfy MTL.

Introduce matrix functions $\widetilde{H}_{k}(t)$ and $\widetilde{U}_{k}(t)$ by the equalities

$$
\widetilde{H}_{k}(t)=J \widetilde{U}(k, t) \widetilde{\eta}(k, t) \widetilde{U}(k, t)^{*} J, \quad \widetilde{U}(k, t)=\widetilde{U}(k-1, t) \widetilde{\xi}(k, t)^{-1},
$$

where $U(0, t)=I_{m}$,

$$
\widetilde{\eta}(k, t)=\left[\begin{array}{ll}
\widetilde{C}(k, t) & 0 \\
0 & 0
\end{array}\right], \quad \widetilde{\xi}(k, t)=\left[\begin{array}{lr}
-i \widetilde{Q}(k, t) & \widetilde{C}(k) \\
\widetilde{C}(k)^{-1} & 0
\end{array}\right] .
$$

Theorem 5.2 Suppose the conditions of Theorem 5.1 are fulfilled. Then the fundamental solutions of the DCS (2.8), where $\widetilde{H}_{k}(t)$ is given by (5.21), in the case $k=2 l$ take the form

$$
\widetilde{w}(k, t, l)=\widetilde{U}(k, t) \widehat{w}_{A}(k, t, l) T D_{l}^{l} T^{-1} \widehat{w}_{A}(0, t, l)^{-1},
$$

and in the case $k=2 l+1$ take the form

$$
\widetilde{w}(k, t, l)=\widetilde{U}(k, t) \widehat{w}_{A}(k, t, l) J\left(I_{m}+i l J \mathcal{P}_{1}\right) T D_{l}^{l} T^{-1} \widehat{w}_{A}(0, t, l)^{-1} .
$$

Here $\widehat{w}_{A}$ is defined in (4.39), matrix functions $\widetilde{C}, \widetilde{Q}, \widehat{\Pi}_{k}$, and $S_{k}$ are defined in (5.12)-(5.18). If $\operatorname{det} A \neq 0$, then we have

$$
\widetilde{U}(k, t)=J^{k} \widehat{w}_{A}(k, t, 0) J^{k} \widehat{w}_{A}(0, t, 0)^{-1} .
$$


The evolution of the corresponding Weyl function for DCS on the semiaxis is given by the formula

$$
\widetilde{\varphi}(t, l)=i\left[\begin{array}{ll}
0 & I_{p}
\end{array}\right] \widehat{w}_{A}(0, t, l) h(l)\left(\left[\begin{array}{ll}
I_{p} & 0
\end{array}\right] \widehat{w}_{A}(0, t, l) h(l)\right)^{-1},
$$

where

$$
h(l)=\left[\begin{array}{c}
I_{p} \\
\frac{l+\sqrt{l^{2}-4}}{2 i} I_{p}
\end{array}\right] \quad\left(l \in \mathbb{C}_{+}\right) .
$$

Proof. According to the first relation in (4.34) and formula (4.37) our matrix functions $\widetilde{U}$ and $\widetilde{\xi}$ given by the formulas (5.21) and (5.22) coincide with those treated in Section 4. Recall that by (2.22) we have $\widetilde{H}_{k}=$ $J w_{0}(k)^{-1} J H_{k} w_{0}(k-1)$. Hence in view of formula (4.33) and the second relation in formula (4.34) we obtain $\widetilde{H}_{k}=J \widetilde{U}(k) \mathcal{P}_{1} \widetilde{U}(k-1)^{-1}$. Notice that $\widetilde{U}$ is $J$-unitary. So according to the first relation in (4.34) we get

$$
\widetilde{H}_{k}(t)=J \widetilde{U}(k, t) \mathcal{P}_{1} \widetilde{\xi}(k, t)^{-1} \widetilde{U}(k, t)^{-1}=J \widetilde{U}(k, t) \mathcal{P}_{1} \widetilde{\xi}(k, t)^{-1} J \widetilde{U}(k, t)^{*} J .
$$

From (5.22) it follows that $\mathcal{P}_{1} \widetilde{\xi}^{-1} J=\widetilde{\eta}$, i.e., (5.28) is equivalent to the first relation in (5.21). In other words our $\widetilde{H}_{k}$ coincides with $\widetilde{H}_{k}$ treated in Section 2 and we can apply Theorem 2.1] Therefore formulas (2.11), (4.34), and (4.38), and normalizing condition $\widetilde{w}(0, t, l)=I_{m}$ yield:

$$
\widetilde{w}(k, t, l)=\widetilde{U}(k, t) \widehat{w}_{A}(k, t, l) U(k, t)^{-1} w(k, t, l) \widehat{w}_{A}(0, t, l)^{-1} .
$$

Taking into account (5.3) and (5.9) we derive now (5.23) and (5.24). Representation (5.25) follows from Remark 2.2.

To derive (5.26) we first need to show that $\varphi(l):=\frac{1}{2}\left(l+\sqrt{l^{2}-4}\right) I_{p}$ is a Weyl function of the initial DCS. Indeed, choosing $K=2 l$, by (15.7) and (5.9) we see that $w(K, l) h(l)=z_{1}^{l} h(l)$. Notice that $i\left(\varphi(l)^{*}-\varphi(l)\right)>0\left(l \in \mathbb{C}_{+}\right)$, i.e., $h(l)^{*} J h(l)>0$. From $w(K, l) h(l)=z_{1}^{l} h(l)$ and $h(l)^{*} J h(l)>0$, using (3.17), we obtain the inequality

$$
\begin{gathered}
h(l)^{*} \sum_{k=0}^{K-1} w(k, l)^{*} H_{k+1} w(k, l) h(l)= \\
\frac{i\left(1-\left|z_{1}\right|^{K}\right)}{l-\bar{l}} h(l)^{*} J h(l)<\frac{i}{l-\bar{l}} h(l)^{*} \operatorname{J} h(l) .
\end{gathered}
$$


By (5.29) the inequality (3.7) for $\varphi(l)=\frac{1}{2}\left(l+\sqrt{l^{2}-4}\right) I_{p}$ is valid, and thus $\varphi$ is a Weyl function for the initial DCS with $H_{k}=J^{k} \mathcal{P}_{2} J^{k}$ on the semiaxis. We apply now Theorem 3.4 and it remains to prove that

$$
\operatorname{det}\left[I_{p} \quad 0\right] \widehat{w}_{A}(0, t, l) h(l) \neq 0 \text {. }
$$

Recall that $h(l)^{*} J h(l)>0$ and $\widehat{w}_{A}(0, t, l)^{*} J \widehat{w}_{A}(0, t, l) \geq J$ for $l \in \mathbb{C}_{+}, l \notin$ $\sigma(A)$. Therefore we have $\widetilde{h}(l)^{*} J \widetilde{h}(l)>0$ for $\widetilde{h}(l)=\widehat{w}_{A}(0, t, l) h(l)$. Suppose there is a vector $f \neq 0$ such that $\left[\begin{array}{ll}I_{p} & 0\end{array}\right] \widetilde{h}(\mu) f=0$ for some $\mu \in \mathbb{C}_{+}, \mu \notin$ $\sigma(A)$. Then, taking into account inequality $f^{*} \widetilde{h}(\mu)^{*} J \widetilde{h}(\mu) f>0$, we see that the $(p+1)$-dimensional subspace $L_{f}=\operatorname{span}\left(h(\mu) f \cup \operatorname{Im}\left[\begin{array}{c}0 \\ I_{p}\end{array}\right]\right)$ is $J$ nonnegative. (Here Im means image and $L_{f}$ is called $J$-nonnegative if for any $g \in L_{f}$ we have $g^{*} J g \geq 0$.) As the maximal dimension of the $J$-nonnegative subspaces is $p$, so we come to a contradiction, i.e., inequality (5.30) is true. Therefore by Theorem 3.4 the last statement of the theorem is true also.

\section{Non-Abelian Toda lattice}

To describe a Bäcklund-Darboux transformation for the NTL (1.3) we introduce $\widehat{\Pi}_{k}(t)$ by the equations (4.10) and (4.11) as before, and introduce an $n \times n$ matrix $B$ and a new set of $m \times n$ matrix functions $\mathrm{E}_{k}(t)$. These matrix functions are given by the initial value $\mathrm{E}_{0}(0)$ and equations

$$
\begin{gathered}
\mathrm{E}_{k}(t)=\xi(k, t) \mathrm{E}_{k-1}(t)+i \mathcal{P}_{1} \mathrm{E}_{k-1}(t) B, \\
\frac{d}{d t} \mathrm{E}_{0}(t)=\mathcal{P}_{1} \mathrm{E}_{0}(t) B-i \psi(0, t) \mathrm{E}_{0}(t) .
\end{gathered}
$$

Under the previous section assumptions we had $B=A^{*}, \mathrm{E}_{k}=i J \widehat{\Pi}_{k}^{*}$. In this section we have five parameter matrices: $A, B, \widehat{\Pi}_{0}(0), \mathrm{七}_{0}(0)$ and $S_{0}(0)$ and assume that the operator identity

$$
A S_{0}(0)-S_{0}(0) B=\widehat{\Pi}_{0}(0) \mathrm{E}_{0}(0)
$$


holds. Now the matrix functions $S_{k}(t)$ are defined by the relations

$$
\begin{gathered}
S_{k}(t)=S_{k-1}(t)-i \widehat{\Pi}_{k-1}(t) \mathcal{P}_{2} \xi(k, t) \mathrm{E}_{k-1}(t) \\
\frac{d}{d t} S_{0}(t)=\frac{1}{2}\left(A S_{0}(t)+S_{0}(t) B-\widehat{\Pi}_{0}(t)\left(\mathcal{P}_{1}-\mathcal{P}_{2}\right) \mathrm{E}_{0}(t)\right) .
\end{gathered}
$$

Then quite similar to the formulas (4.19), (4.21), and (4.26) we derive

$$
\begin{gathered}
A S_{k}(t)-S_{k}(t) B=\widehat{\Pi}_{k}(t) \mathrm{Ł}_{k}(t) \\
\frac{d}{d t} \mathrm{Ł}_{k}(t)=\mathcal{P}_{1} \mathrm{Ł}_{k}(t) B-i \psi(k, t) \mathrm{£}_{k}(t) \\
\frac{d}{d t} S_{k}(t)=\frac{1}{2}\left(A S_{k}(t)+S_{k}(t) B-\widehat{\Pi}_{k}(t)\left(\mathcal{P}_{1}-\mathcal{P}_{2}\right) \mathrm{E}_{k}(t)\right) .
\end{gathered}
$$

Finally put

$$
\begin{gathered}
\widetilde{C}(k, t)=C(k, t)-i Z_{12}(k-1, t) \quad(k>0), \\
\widetilde{C}(0, t)=\left(C(0, t)^{-1}+i Z_{21}(0, t)\right)^{-1}, \\
\widetilde{Q}(k, t)=Q(k, t)+Z_{11}(k-1, t)-Z_{11}(k, t) \quad(k>0),
\end{gathered}
$$

where $Z_{i j}(k)$ are $p \times p$ blocks given by the formulas

$$
Z(k)=\left\{Z_{i j}(k)\right\}_{i, j=1}^{2}:=\mathrm{Ł}_{k} S_{k}^{-1} \widehat{\Pi}_{k} .
$$

The Bäcklund-Darboux transformation result is given by the following generalization of Theorem 4.1.

Theorem 6.1 Suppose matrix functions $C(k, t)$ and $Q(k, t)$ satisfy $N T L$ (1.3) and $C(0, t)$ is continuous. Suppose additionally that matrix functions $C(0, t)^{-1}+i Z_{21}(0, t)$ and matrix functions $S_{k}(t)$ given by 6.4), (6.5), and (6.12) are invertible in the domain $-\varepsilon_{1}<t<\varepsilon_{2}$, $0 \leq k<K$. Then the matrix functions $\widetilde{C}$ and $\widetilde{Q}$ are well defined by (6.9)-(6.11) and satisfy NTL in the domain $-\varepsilon_{1}<t<\varepsilon_{2}, 0<k<K$. 
Pr o of. The proof is similar to the proof of Theorem 4.1. We shall need the evident identities:

$$
\mathcal{P}_{2} \xi(k)=\xi(k)^{-1} \mathcal{P}_{1}=\mathcal{P}_{2} \xi(k) \mathcal{P}_{1}, \quad \xi(k)^{-1} \mathcal{P}_{1} \xi(k) \mathcal{P}_{2}=\mathcal{P}_{2} \xi(k)^{2} \mathcal{P}_{2}=\mathcal{P}_{2}
$$

From (6.4) it follows that

$$
S_{k}^{-1}=S_{k-1}^{-1}+i S_{k}^{-1} \widehat{\Pi}_{k-1} \mathcal{P}_{2} \xi(k) \mathrm{E}_{k-1} S_{k-1}^{-1} .
$$

Using (6.1), (6.14), and the first equality in relations (6.13) by induction we can show that

$$
\mathrm{E}_{k}(t) S_{k}(t)^{-1}=\widetilde{\xi}(k, t) \mathrm{E}_{k-1}(t) S_{k-1}(t)^{-1}+i \mathcal{P}_{1} \mathrm{E}_{k-1}(t) S_{k-1}(t)^{-1} A,
$$

where

$$
\widetilde{\xi}(k, t):=\xi(k, t)-i \mathcal{P}_{1} Z(k-1, t)+i Z(k, t) \mathcal{P}_{1} .
$$

We cannot use $J$-unitary matrices to derive the equality

$$
\widetilde{C}(k, t)^{-1}=C(k, t)^{-1}+i Z_{21}(k, t),
$$

which is the analog of the important formula (4.32), anymore. Therefore we shall consider now $\mathcal{P}_{2} \widetilde{\xi}(k)^{2} \mathcal{P}_{2}$ for $k>0$. (If $k=0$, then formula (6.17) follows from (6.10).) By (6.13) and (6.16) we derive

$$
\mathcal{P}_{2} \widetilde{\xi}(k)^{2} \mathcal{P}_{2}=\mathcal{P}_{2} \widetilde{\xi}(k) \mathcal{P}_{1}(\xi(k)-i Z(k-1)) \mathcal{P}_{2} .
$$

Notice further that according to (4.10) and (6.13) we have

$$
\mathcal{P}_{2} Z(k) \mathcal{P}_{1} \xi(k) \mathcal{P}_{2}=\mathcal{P}_{2} \mathrm{E}_{k} S_{k}^{-1} \widehat{\Pi}_{k-1} \mathcal{P}_{2}
$$

Hence taking into account formula (6.15) and equality $\mathcal{P}_{2} \widetilde{\xi}(k)=\mathcal{P}_{2} \widetilde{\xi}(k) \mathcal{P}_{1}$ we get

$$
\mathcal{P}_{2} Z(k) \mathcal{P}_{1} \xi(k) \mathcal{P}_{2}=\mathcal{P}_{2} \tilde{\xi}(k) \mathcal{P}_{1} Z(k-1) \mathcal{P}_{2}
$$

Using equalities (6.13) and (6.16) by (6.18) and (6.20) we obtain

$$
\mathcal{P}_{2} \tilde{\xi}(k)^{2} \mathcal{P}_{2}=\mathcal{P}_{2} \tilde{\xi}(k) \mathcal{P}_{1} \xi(k) \mathcal{P}_{2}-i \mathcal{P}_{2} Z(k) \mathcal{P}_{1} \xi(k) \mathcal{P}_{2}=\mathcal{P}_{2}
$$


In view of the formulas (6.9) and (6.16) equality (6.21) yields (6.17). Moreover $\widetilde{\xi}$ preserves the form (4.37) from the previous section.

The transfer matrix function $\widehat{w}_{A}$ is given here by the formula

$$
\widehat{w}_{A}(k, t, l)=I_{m}-\mathrm{E}_{k}(t)^{*} S_{k}(t)^{-1}\left(A-l I_{n}\right)^{-1} \widehat{\Pi}_{k}(t) .
$$

From (4.2), (4.10), (6.13), and (6.22) it follows that

$$
\begin{aligned}
\widehat{w}_{A}(k, l) G(k, l)= & -l \mathcal{P}_{1}+i \xi(k)-i \mathrm{E}_{k} S_{k}^{-1}\left(A-l I_{n}\right)^{-1} \widehat{\Pi}_{k-1} \\
& -\mathrm{E}_{k} S_{k}^{-1} \widehat{\Pi}_{k-1} \xi(k)^{-1} \mathcal{P}_{1} .
\end{aligned}
$$

Taking into account (6.15) we rewrite (6.23):

$$
\begin{aligned}
\widehat{w}_{A}(k, l) G(k, l)= & -l \mathcal{P}_{1}+i \xi(k)-Z(k) \mathcal{P}_{1}+i \widetilde{\xi}(k)\left(\widehat{w}_{A}(k-1, l)-I_{m}\right) \\
& +\mathcal{P}_{1} \mathrm{E}_{k-1} S_{k-1}^{-1} A\left(A-l I_{n}\right)^{-1} \widehat{\Pi}_{k-1} .
\end{aligned}
$$

Finally using the equality $A=\left(A-l I_{n}\right)+l I_{n}$ and definitions (4.44) and (6.16) of $\widetilde{G}$ and $\widetilde{\xi}$, respectively, we obtain

$$
\widehat{w}_{A}(k, t, l) G(k, t, l)=\widetilde{G}(k, t, l) \widehat{w}_{A}(k-1, t, l) .
$$

The proof of (4.41), where $F$ and $\widetilde{F}$ are defined by (4.42), and $\widetilde{\psi}$ is defined by (4.29), (6.9) and (6.10), is quite analogous to the one in Section 3. After we recall that NTL is equivalent to the zero curvature equation (4.1) it remains to derive from (4.41) and (6.25) the equation (4.48) and then (4.49). These arguments coincide with the corresponding arguments from Theorem 4.1.

Finally notice that the important case of blow-up of the NTL solution can be studied in terms of the invertibility of $S_{k}(t)$.

\section{Appendix: Jacobi matrices}

DCS are closely related to the well-known Jacobi matrices. For symmetric block Jacobi matrices we shall obtain an analog of Theorem 3.5. Suppose 
that the sets of matrices $\{C(k)\}_{k \geq 0}$ and $\{Q(k)\}_{k>0}$ such that

$$
C(k) Q(k)^{*}=Q(k) C(k) \quad(k>0), \quad C(k)>0 \quad(k \geq 0)
$$

are given. Fix $n>0$, two $n \times n$ parameter matrices $A$ and $S_{0}$ and $n \times m$ parameter matrix $\widehat{\Pi}_{0}$, such that

$$
A S_{0}-S_{0} A^{*}=i \widehat{\Pi}_{0} J \widehat{\Pi}_{0}^{*}, \quad S_{0}>0 .
$$

Introduce matrices $\widehat{\Pi}_{k}, S_{k}, \widetilde{C}(k)$, and $\widetilde{Q}(k)$ by the same equalities as in Section 4 but without dependence on $t$ :

$$
\begin{gathered}
\widehat{\Pi}_{k}=\widehat{\Pi}_{k-1} \xi(k)^{-1}-i A \widehat{\Pi}_{k-1} \mathcal{P}_{2}, \quad S_{k}=S_{k-1}+\widehat{\Pi}_{k-1} \zeta(k) \widehat{\Pi}_{k-1}^{*}, \\
\xi(k)=\left[\begin{array}{rr}
-i Q(k) & C(k) \\
C(k)^{-1} & 0
\end{array}\right], \quad \zeta(k)=\left[\begin{array}{rr}
0 & 0 \\
0 & C(k)^{-1}
\end{array}\right], \quad(7.3) \\
\widetilde{C}(k)=C(k)+X_{22}(k-1) \quad(k>0), \quad \widetilde{C}(0, t)=\left(C(0)-X_{11}(0)\right)^{-1}, \quad(7.5) \\
\widetilde{Q}(k)=Q(k)+i\left(X_{21}(k-1)-X_{21}(k)\right), \quad X(k)=\left\{X_{i j}\right\}_{i, j=1}^{2}=\widehat{\Pi}_{k}^{*} S_{k}^{-1} \widehat{\Pi}_{k} .
\end{gathered}
$$

Introduce now a block Jacobi matrix by the equalities

$$
\widetilde{L}=\left[\begin{array}{cccccc}
\widetilde{b}_{1} & \widetilde{a}_{1} & 0 & 0 & 0 & \ldots \\
\widetilde{c}_{2} & \widetilde{b}_{2} & \widetilde{a}_{2} & 0 & 0 & \ldots \\
0 & \widetilde{c}_{3} & \widetilde{b}_{3} & \widetilde{a}_{3} & 0 & \ldots \\
\ldots & \ldots & \ldots & \ldots & \ldots & \ldots
\end{array}\right]
$$

where for $k>0$ we put

$$
\widetilde{a}_{k}=-i \widetilde{C}(k)^{-\frac{1}{2}} \widetilde{C}(k+1)^{\frac{1}{2}}, \quad \widetilde{b}_{k}=\widetilde{b}_{k}^{*}=\widetilde{C}(k)^{-\frac{1}{2}} \widetilde{Q}(k) \widetilde{C}(k)^{\frac{1}{2}}, \quad \widetilde{c}_{k}=\widetilde{a}_{k-1}^{*} .
$$

Matrix $\widetilde{L}$ can be considered as the GBDT of the Jacobi matrix $L$ defined by the same formulas (17.7) and (7.8) but with tildes removed. 
Theorem 7.1 Suppose Jacobi matrix $\widetilde{L}$ is given by the formulas (7.3)-(7.8) and relations (7.1) and (7.2) are valid. If $f \neq 0$ is an eigenvector of $A$ such that

$$
A f=\mu f, \quad\left[\begin{array}{ll}
I_{p} & 0
\end{array}\right] \widehat{\Pi}_{0}^{*} S_{0}^{-1} f=0,
$$

then the vector $Y=\operatorname{col}\left[y_{1} y_{2} \ldots\right]$, where col means column and

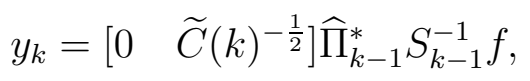

is an eigenvector of $\widetilde{L}$ :

$$
\widetilde{L} Y=\mu Y, \quad \sum_{k=1}^{\infty} y_{k}^{*} y_{k}<\infty .
$$

Pro of. First notice that the equality $\widetilde{b}_{k}=\widetilde{b}_{k}^{*}$ in (17.8) follows from (17.1). Next similar to the proof of the equation (6.15) we shall show that

$$
\widehat{\Pi}_{k}^{*} S_{k}^{-1}=i \mathcal{P}_{2} \widehat{\Pi}_{k-1}^{*} S_{k-1}^{-1} A+\left[\begin{array}{ll}
0 & \widetilde{C}(k)^{-1} \\
\widetilde{C}(k) & -i \widetilde{Q}(k)
\end{array}\right] \widehat{\Pi}_{k-1}^{*} S_{k-1}^{-1}
$$

Indeed, by (1.2), (2.2), (2.14), and (4.9) we get

$$
\begin{gathered}
\widehat{\Pi}_{k}^{*} S_{k}^{-1}=i U(k)^{*} H_{k} J\left(U(k-1)^{*}\right)^{-1} \widehat{\Pi}_{k-1}^{*} S_{k-1}^{-1} A+U(k)^{*}\left(I_{m}+\right. \\
\left.H_{k} J \Pi_{k-1}^{*} S_{k-1}^{-1} \Pi_{k-1} J-\Pi_{k}^{*} S_{k}^{-1} \Pi_{k} J H_{k} J\right)\left(U(k-1)^{*}\right)^{-1} \widehat{\Pi}_{k-1}^{*} S_{k-1}^{-1} .
\end{gathered}
$$

By (4.5)-(4.7) we rewrite (7.13) as

$$
\begin{gathered}
\widehat{\Pi}_{k}^{*} S_{k}^{-1}=i J \eta(k)\left(\xi(k)^{-1}\right)^{*} \widehat{\Pi}_{k-1}^{*} S_{k-1}^{-1} A+\left(\left(\xi(k)^{-1}\right)^{*}+\right. \\
\left.J \eta(k)\left(\xi(k)^{-1}\right)^{*} \widehat{\Pi}_{k-1}^{*} S_{k-1}^{-1} \widehat{\Pi}_{k-1} J-\widehat{\Pi}_{k}^{*} S_{k}^{-1} \widehat{\Pi}_{k} \eta(k)\left(\xi(k)^{-1}\right)^{*}\right) \widehat{\Pi}_{k-1}^{*} S_{k-1}^{-1} .
\end{gathered}
$$

As $J \eta(k)\left(\xi(k)^{-1}\right)^{*}=\mathcal{P}_{2}$, so (17.14) yields

$$
\widehat{\Pi}_{k}^{*} S_{k}^{-1}=i \mathcal{P}_{2} \widehat{\Pi}_{k-1}^{*} S_{k-1}^{-1} A+
$$




$$
\left[\begin{array}{lr}
0 & C(k)^{-1} \\
C(k) & -i C(k) Q(k)^{*} C(k)^{-1}
\end{array}\right]+\left[\begin{array}{lr}
0 & -X_{11}(k) \\
X_{22}(k-1) & X_{21}(k-1)-X_{21}(k)
\end{array}\right] .
$$

Finally, using (4.32), (7.1), (7.4)-(7.6), we transform (7.15) into (7.12).

Now, according to the first relations in (77.8) and (17.9) and to the formulas (17.10) and (7.12) we obtain

$$
\begin{gathered}
\widetilde{a}_{k} y_{k+1}=-i \widetilde{C}(k)^{-\frac{1}{2}}\left[\begin{array}{ll}
0 & I_{p}
\end{array}\right] \widehat{\Pi}_{k}^{*} S_{k}^{-1} f= \\
\mu y_{k}-\widetilde{C}(k)^{-\frac{1}{2}} \widetilde{Q}(k) \widetilde{C}(k)^{\frac{1}{2}} y_{k}-i \widetilde{C}(k)^{\frac{1}{2}}\left[I_{p} \quad 0\right] \widehat{\Pi}_{k-1}^{*} S_{k-1}^{-1} f .
\end{gathered}
$$

Using the second relation in the definition (7.8) we rewrite (7.16) as

$$
\widetilde{b}_{k} y_{k}+\widetilde{a}_{k} y_{k+1}=\mu y_{k}-i \widetilde{C}(k)^{\frac{1}{2}}\left[\begin{array}{ll}
I_{p} & 0
\end{array}\right] \widehat{\Pi}_{k-1}^{*} S_{k-1}^{-1} f .
$$

In particular, in view of the second relation in (7.9) formula (7.17) yields

$$
\widetilde{b}_{1} y_{1}+\widetilde{a}_{1} y_{2}=\mu y_{1}
$$

For $k>1$ from (7.12) we derive

$$
\begin{gathered}
i \widetilde{C}(k)^{\frac{1}{2}}\left[\begin{array}{cc}
I_{p} & 0
\end{array}\right] \widehat{\Pi}_{k-1}^{*} S_{k-1}^{-1} f= \\
i \widetilde{C}(k)^{\frac{1}{2}} \widetilde{C}(k-1)^{-\frac{1}{2}}\left[\begin{array}{ll}
0 & \left.\widetilde{C}(k-1)^{-\frac{1}{2}}\right] \widehat{\Pi}_{k-2}^{*} S_{k-2}^{-1} f=\widetilde{a}_{k-1}^{*} y_{k-1} .
\end{array}\right.
\end{gathered}
$$

By (17.17) and (7.19) we get

$$
\widetilde{c}_{k} y_{k-1}+\widetilde{b}_{k} y_{k}+\widetilde{a}_{k} y_{k+1}=\mu y_{k} \quad(k>1) .
$$

Formulas (7.18) and (7.20) yield the first relation in (7.11).

To prove the second relation in (7.11) consider expressions $y_{k}^{*} y_{k}$. One can see that

$$
y_{k}^{*} y_{k}=f^{*} S_{k-1}^{-1} \widehat{\Pi}_{k-1} \widetilde{\zeta}(k) \widehat{\Pi}_{k-1}^{*} S_{k-1}^{-1} f, \quad \widetilde{\zeta}(k)=\left[\begin{array}{rr}
0 & 0 \\
0 & \widetilde{C}(k)^{-1}
\end{array}\right] .
$$


By (4.9) we rewrite (7.21) in the form

$$
y_{k}^{*} y_{k}=g_{k-1}^{*} w_{0}(k-1)^{*} J U(k-1) \widetilde{\zeta}(k) U(k-1)^{*} J w_{0}(k-1) g_{k-1},
$$

where $g_{k}$ are defined in (3.22). Hence, using (4.34) and the fact that $w_{0}(k)$ is $J$-unitary, we obtain

$$
y_{k}^{*} y_{k}==g_{k-1}^{*} J \widetilde{U}(k-1) \widetilde{\zeta}(k) \widetilde{U}(k-1)^{*} J g_{k-1} .
$$

As $\widetilde{\xi}(k) \widetilde{\zeta}(k) \widetilde{\xi}(k)^{*}=\widetilde{\eta}(k)$, formulas (5.21) and (7.23) yield:

$$
y_{k}^{*} y_{k}=g_{k-1}^{*} J \widetilde{U}(k) \widetilde{\xi}(k) \widetilde{\zeta}(k) \widetilde{\xi}(k)^{*} \widetilde{U}(k)^{*} J g_{k-1}=g_{k-1}^{*} \widetilde{H}_{k} g_{k-1} .
$$

It was shown in Theorem 3.5 that $\sum_{k=1}^{\infty} g_{k-1}^{*} \widetilde{H}_{k} g_{k-1}<\infty$, i.e., by (17.24) the second relation in (7.11) is true.

\section{References}

[1] M.J. Ablowitz and J.F. Ladik 1976, Nonlinear differential-difference equations and Fourier analysis, J. Math. Phys. 17, 1011-1018

[2] M.J. Ablowitz and H. Segur 1981 "Solitons and the inverse scattering transform" (Philadelphia: SIAM Stud. Appl. Math. 4)

[3] Yu.M. Berezanskij 1986 The integration of semi-infinite Toda chain by means of inverse spectral problem, Rep. Math. Phys. 24:1, 21-47

[4] M. Bruschi, S.V. Manakov, O. Ragnisco, and D. Levi 1980 The nonabelian Toda lattice - Discrete analogue of the matrix Schrodinger spectral problem, J. Math. Phys. 21, 2749-2753

[5] M. Bruschi and O. Ragnisco 1988 Recursion operator and Bäcklund transformations for the Ruijsenaars-Toda lattice, Phys. Lett. A 129:1, 21-25

[6] W. Bulla, F. Gesztesy, H. Holden, and G. Teschl 1998 Algebrogeometric quasi-periodic finite-gap solutions of the Toda and Kacvan Moerbeke hierarchies, Mem. Am. Math. Soc. 641, 1-79

[7] F. Calogero and A. Degasperis 1982 "Spectral transform and solitons" (Amsterdam: North-Holland) 
[8] D.V. Chudnovsky and G.V. Chudnovsky 1982 Bäcklund transformation as a method of decomposition and reproduction of twodimensional nonlinear systems, Phys. Lett. A 87:7, 325-329

[9] S. Clark and F. Gesztesy, On Weyl-Titchmarsh theory for singular finite difference Hamiltonian systems (arXiv: math.SP/0312177)

[10] L. de Branges 1968 "Hilbert spaces of entire functions" ( N.J.: Prentice-Hall)

[11] P.A. Deift 1978 Applications of a commutation formula, Duke Math. J. 45, 267-310

[12] J.C. Eilbeck and M. Johansson, The discrete nonlinear Schrödinger equation - 20 years on (arXiv:nlin.PS/0211049)

[13] P. Etingof, I. Gelfand and V. Retakh 1998 Nonabelian integrable systems, quasideterminants, and Marchenko lemma, Math. Res. Lett. 5:1-2, 1-12

[14] L.D. Faddeev and L.A. Takhtajan 1986 "Hamiltonian methods in the theory of solitons" (NY: Springer-Verlag)

[15] L. Faybusovich and M. Gekhtman 2000 Elementary Toda orbits and integrable lattices, J. Math. Phys. 41:5, 2905-2921

[16] M. Gekhtman 1998 Hamiltonian structure of non-Abelian Toda lattice, Lett. Math. Phys. 46:3, 189-205

[17] F. Gesztesy, W. Schweiger, and B. Simon 1991 Commutation methods applied to the mKdV-equation, Trans. Amer. Math. Soc. 324, 465-525

[18] I. Gohberg, M.A. Kaashoek, and A.L. Sakhnovich 2001 Bound states for canonical systems on the half and full line: explicit formulas, IEOT 40:3, 268-277

[19] I. Gohberg, M.A. Kaashoek, and A.L. Sakhnovich 2002 Scattering problems for a canonical system with a pseudo-exponential potential, Asymptotic Analysis 29:1, 1-38

[20] I. Gohberg and M.G. Krein 1970 "Theory and applications of Volterra operators in Hilbert space" (Transl. of math. monographs 24, Providence, Rhode Island)

[21] M. Kac and P. van Moerbeke 1975 A complete solution of the periodic Toda problem, Proc. Natl. Acad. Sci. USA 72, 2879-2880

[22] R.E. Kalman, P. Falb and M. Arbib 1969 "Topics in mathematical system theory" (NY: McGraw-Hill) 
[23] I.M. Krichever 1981 The periodic non-Abelian Toda chain and its two-dimensional generalization, Russ.Math.Surv. 36:2, 82-89

[24] I.M. Krichever and K. Vaninsky, The periodic and open Toda lattice, (arXiv:hep-th/0010184)

[25] V.B. Kuznetsov, M. Salerno, and E.K. Sklyanin 2000 Quantum Bäcklund transformation for the integrable DST model, J. Phys. A, Math. Gen. 33:1, 171-189

[26] D. Levi and O. Ragnisco (eds) 2000 SIDE III - Symmetries and integrability of difference equations, CRM Proceedings and Lecture Notes 25 (Providence, RI: AMS)

[27] M.S. Livšic 1960 On a class of linear operators in Hilbert space, Amer. Math. Soc. Transl. (2) 13, 85-103

[28] V. A. Marchenko 1988 "Nonlinear equations and operator algebras" (Dordrecht: Reidel)

[29] V.B. Matveev and M.A. Salle 1991 "Darboux transformations and solitons" (Berlin: Springer-Verlag)

[30] P.D. Miller, A.C. Scott, J. Carr, and J.C. Eilbeck 1991 Binding energies for discrete nonlinear Schrodinger equations, Phys. Scr. 44:6, 509-516

[31] R. Miura (ed.) 1976 "Bäcklund Transformations," Lecture Notes in Math. 515 (Berlin: Springer-Verlag)

[32] J. Rovnyak and L. A. Sakhnovich 2002 Some indefinite cases of spectral problems for canonical systems of difference equations, Lin.Alg.Appl. 343-344, 267-289

[33] A.L. Sakhnovich 1993 Exact solutions of nonlinear equations and the method of operator identities, Lin.Alg.Appl. 182, 109-126

[34] A.L. Sakhnovich 1994 Dressing procedure for solutions of nonlinear equations and the method of operator identities, Inverse problems 10, 699-710

[35] A.L. Sakhnovich 1997 Iterated Bäcklund-Darboux transform for canonical systems, J. Functional Anal. 144, 359-370

[36] A.L. Sakhnovich 2001 Generalized Bäcklund-Darboux transformation: spectral properties and nonlinear equations, J. Math. Anal. Appl. 262, 274-306

[37] A.L. Sakhnovich 2003 Dirac type system on the axis: explicit formulas for matrix potentials with singularities and soliton-positon interactions, Inverse Problems 19, 845-855 
[38] A.L. Sakhnovich 2003 Non-Hermitian matrix Schrödinger equation: Bäcklund-Darboux transformation, Weyl functions, and $\mathcal{P} \mathcal{T}$ symmetry, J. Phys. A 36, 7789-7803

[39] A.L. Sakhnovich and J.P. Zubelli 2001 Bundle bispectrality for matrix differential equations, IEOT 41, 472-496

[40] L.A. Sakhnovich 1976 On the factorization of the transfer matrix function, Sov. Math. Dokl. 17, 203-207

[41] L.A. Sakhnovich 1997 " Interpolation theory and its applications" (Dordrecht: Kluwer Academic Publishers)

[42] L.A. Sakhnovich 1999 "Spectral theory of canonical differential systems, method of operator identities," OT 107 (Basel: BirkhäuserVerlag)

[43] C. Schiebold 1998 An operator-theoretic approach to the Toda lattice equation, Physica D 122:1-4, 37-61

[44] G. Teschl 2001 Almost everything you always wanted to know about the Toda equation, Jahresber. Dtsch. Math.-Ver. 103:4, 149-162

[45] M. Toda 1989 "Nonlinear waves and solitons. Mathematics and Its Applications" (Dordrecht: Kluwer Academic Publishers)

[46] K.L. Vaninsky 2003 The Atiyah-Hitchin bracket and the open Toda lattice, J. Geom. Phys. 46:3-4, 283-307

[47] V.E.Zakharov and A.V.Mikhailov 1980 On the integrability of classical spinor models in two-dimensional space-time, Comm. Math. Phys. 74, 21-40 